\title{
Monetary Policy in a Channel System*
}

\author{
Aleksander Berentsen \\ Department of Economics, University of Basel \\ Cyril Monnet \\ DG-Research, European Central Bank
}

July 4, 2007

\begin{abstract}
Channel systems for conducting monetary policy are becoming increasingly popular. Despite their popularity, the consequences of implementing policy with a channel system are not well understood. We develop a general equilibrium framework of a channel system and investigate the optimal policy. A novel aspect of the channel system is that a central bank can "tighten" or "loosen" its policy without changing its target rate by increasing the interest-rate spread symmetrically around the target rate. This questions the characterization of optimal policy through interest-rate rules, as done in a large body of the literature on the optimal design of interest-rate rules.
\end{abstract}

JEL: E40, E50, D83

Keywords: Monetary Policy, Interest Rates, Search.

\footnotetext{
${ }^{*}$ We have received useful comments from the participants of several seminars and workshops. We are especially grateful to Vitor Gaspar, Ilhyock Shim, Neil Wallace, Christopher Waller, Michael Woodford and Randall Wright for their comments. Berentsen thanks the Federal Reserve Bank of Cleveland, the European Central Bank and the University of Pennsylvania for research support. The views expressed herein are those of the authors and not those of the European Central Bank or the Eurosystem.
} 


\section{Introduction}

Channel systems for conducting monetary policy are becoming increasingly popular. Several central banks already use a channel system, and others are using at least some features of the channel system. ${ }^{1}$ Despite its popularity, the consequences of implementing monetary policy with a channel system are not well understood. How does implementation of monetary policy in a channel system differ from plain-vanilla open market operations? Given the use of a channel system, how should interest rates be set to maximize welfare? The purpose of this paper is to study the theoretical properties of a channel system.

In a channel system, a central bank offers two standing facilities: a lending facility where it is ready to supply money overnight at a given lending rate against collateral and a deposit facility where banks can make overnight deposits to earn a deposit rate. The interest-rate corridor is chosen to keep the overnight interest rate in the money market close to the target rate. In a pure channel system, a change in policy is implemented by simply changing the corridor without any open market operations.

There are several stylized facts of channel systems that a reasonable theoretical model has to explain. First, all central banks set a strictly positive interest-rate spread - defined as the difference between the lending and the deposit rates. Second, central banks typically react to changing economic conditions by increasing or decreasing their interest-rate corridor without changing its spread. Third, the money market rate tends to be in the middle of the corridor. We construct a general equilibrium model that explains these stylized facts. Moreover, we shed some light on the following questions. First, why do central banks choose different corridors? Most central banks choose a corridor of 50 basis points (e.g. Australia, Canada and New Zealand), while

\footnotetext{
${ }^{1}$ For example, versions of a channel system are operated by the Bank of Canada, Bank of England, the European Central Bank, the Reserve Bank of Australia, and the Reserve Bank of New Zealand. The US Federal Reserve System recently modified the operating procedures of its discount window facility in a way that it now shares elements of a standing facility. Prior to 2003, the discount window rate was set below the target federal fund rate, but banks faced penalties when accessing the discount window. In 2003, the Federal Reserve decided to set the discount window rate 100 basis points above the target federal fund rate and eased access conditions to the discount window. The resulting framework is similar to a channel system, where the deposit rate is zero and the lending rate 100 basis point above the target rate.
} 
the European Central Bank's (ECB) lending rate is 200 basis points higher than its deposit rate (Figure 1). ${ }^{2}$ Second, why can some central banks control the overnight interest rate very tightly while others cannot? For instance, the overnight interbank cash rate in New Zealand is almost always on the policy rate set by the Reserve Bank (Figure 2). In contrast, the uncollateralized Euro overnight rate and the short-term collateralized Euro repo rate fluctuate considerably around the minimum bid rate set by the ECB (Figure 1).

Figure 1: Interest-rate channel of the European Central Bank EONIA (Euro OverNight Index Average) and Eurepo (reference rate for the Euro GC repo market) Source: European Banking Federation and ECB

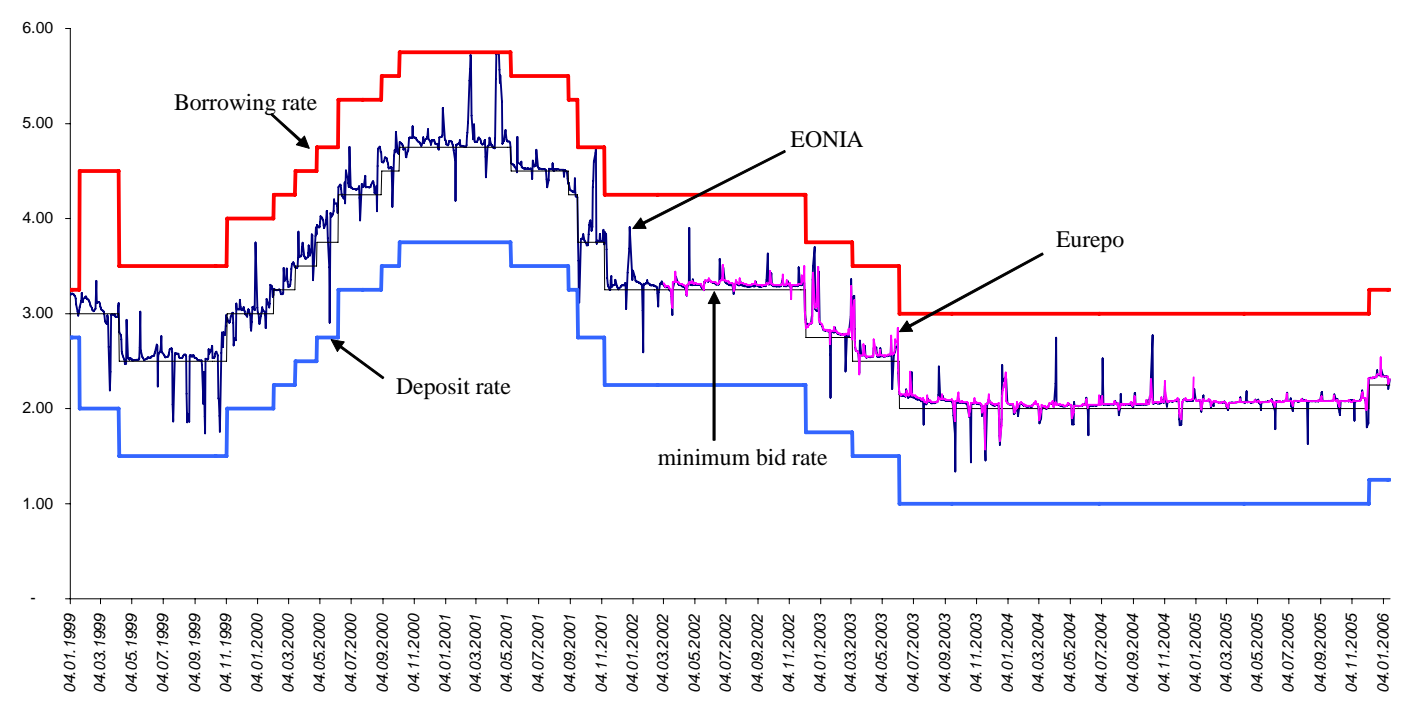

To study the stylized facts and questions, we construct a dynamic general equilibrium model of a channel system with a money market and a welfare-optimizing central bank. Households are subject to idiosyncratic trading shocks which generate random liquidity needs, where a positive liquidity shock for one household corresponds to a negative liquidity shock for another household. ${ }^{3}$ The shocks can be partially insured

\footnotetext{
${ }^{2}$ As can be seen from Figure 1, the ECB increased its spread dramatically from 50 basis points to 250 basis points around February 1999 before reducing it to 200 basis points around April 1999.

${ }^{3}$ We abstract from modeling commercial banks explicitly. Rather, we assume that households have direct access to the money market and the central bank's lending and deposit facility. The trading shocks are an approximation for liquidity shocks faced by commercial banks after trading in the money market. Since there is no feasible trading of reserves after this market has closed, banks which need liquidity have no choice but to use the standing facility offered by the central bank.
} 
in a secured money market. To provide further insurance, the central bank operates a standing facility where households can borrow or deposit money at the specified rates. In accordance with central bank practice, there is no limit to the size of deposits on which interest is paid, and there is no limit to the size of a loan that a household can obtain provided that the loan is fully collateralized. Finally, the cost of pledging collateral is explicit and money is essential. ${ }^{4}$

Within this framework we answer the following three questions. First, what is the optimal interest-rate corridor? Second, what is the optimal collateral policy? Third, how does a change of the corridor affect the money market rate?

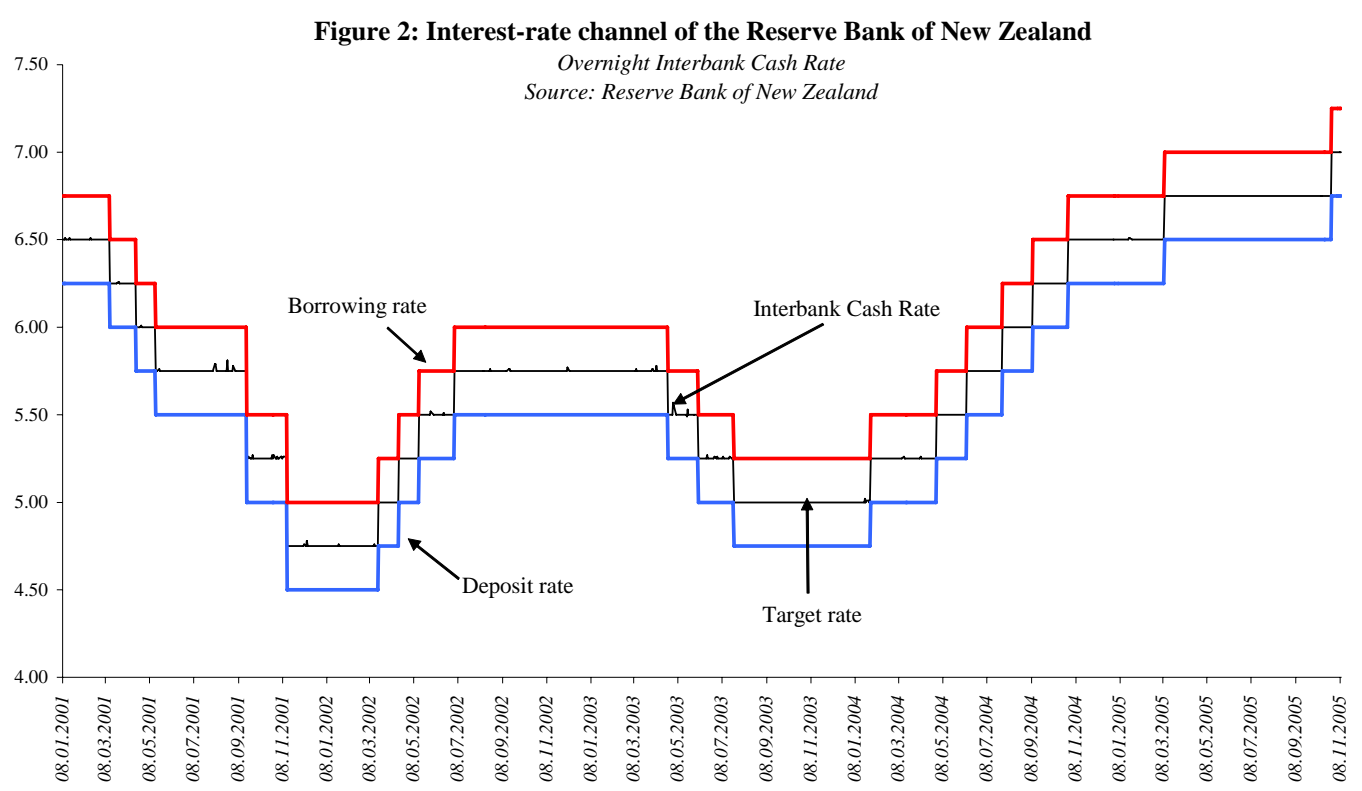

The following results emerge from our model. First, it is optimal to have a positive spread if the opportunity cost of holding collateral is positive. ${ }^{5}$ Second, the optimal spread is decreasing in the rate of return of the collateral and equal to zero when the opportunity cost of acquiring collateral is zero. Third, a central bank has two

\footnotetext{
${ }^{4} \mathrm{By}$ 'essential,' we mean that the use of money expands the set of allocations (Kocherlakota 1998 and Wallace 2001).

${ }^{5}$ The rate of return of the collateral determines the opportunity costs for commercial banks of accessing the lending facility of the central bank where a high rate of return implies a small or zero opportunity cost. Assets accepted as collateral are typically low-risk and low-yield assets such as government securities.
} 
equivalent options for implementing a given policy: it can either shift the corridor while keeping the interest-rate spread constant, or it can change the spread. For instance, to change its policy, it can keep the deposit rate constant and only change the borrowing rate as done, for example, by the US Federal Reserve System or it can shift the corridor without changing its spread as done by the European Central Bank. Fourth, we also find that the money market rate is above the target rate if the opportunity cost of holding collateral is positive. ${ }^{6}$

A very interesting aspect of the channel system is that a central bank can "tighten" or "loosen" its policy without changing its target rate. The reason is that, by increasing the spread of the corridor symmetrically around the target rate, the central bank worsens the option for banks of accessing the standing facility. As a result, the policy regime is tighter. ${ }^{7}$ More generally, this result suggests that a characterization of policy through an interest-rate rule, as it is commonly done in a large body of the literature, is incomplete. Rather, in a channel system, any policy must be characterized through an interest-rate corridor rule. We provide more discussion on this result in the literature section below.

Literature There are very few theoretical studies of channel systems, and all of them are partial equilibrium models. An early contribution is the model of reserve management under uncertainty by Poole (1968). Woodford (2000, 2001, 2003) discusses and analyses the channel system to address the question of how to conduct monetary policy in a world with a vanishing stock of money. Whitesell (2006) evaluates reserves regimes versus channel systems. Elements of channel systems have been previously described in Gaspar, Quiros and Mendizabal (2004), Guthrie and Wright (2000), and Heller and Lengwiler (2003).

It appears that there are two reasons why there is no other general equilibrium analysis of a channel system. First, money growth is endogenous in such a system. In

\footnotetext{
${ }^{6}$ This property of the model provides a rationale for the observation that the collateralized Eurepo rate tends to be above the minimum bid rate and very close to the uncollateralized EONIA rate (Figure 1). Our model suggests that the ECB can get the money market rate closer to its minimum bid rate if it allows for less costly collateral.

${ }^{7}$ This result suggests that the ECB with its 200-basis-points corridor implements a tighter monetary policy than the other central banks operating a channel system mentioned before.
} 
contrast, most theoretical models of monetary policy characterize optimal policy in terms of a path for the money supply. In practice, however, monetary policy involves rules for setting nominal interest rates, and most central banks specify operating targets for overnight interest rates. ${ }^{8}$ This paper, therefore, is an attempt to break the apparent dichotomy (Goodhart, 1989) between theoretical analysis and central bank practices.

The second reason is related to the widespread belief that modeling the details of the framework used to implement a given interest-rate rule is unimportant when analyzing optimal monetary policy. That is, it is taken for granted that the economic consequences of interest-rate rules do not hinge on the specific details of monetary policy implementation. However, our general equilibrium analysis reveals that a characterization of optimal policy and its implementation cannot be separated. To see this, consider any interest-rate rule in a system with zero deposit rate as operated, for example, by the US Federal Reserve System. Such an interest-rate rule uniquely determines how "tight" or "loose" the policy is. In contrast, the same rule or any other interest-rate rule has no meaning in a channel system since it does not determine whether a policy is "tight" or "loose." Consequently, in a channel system optimal policy must not only state an interest-rate rule but it must state an interest-rate corridor rule. This is a new insight, which goes beyond what we already know from the large and growing body of literature on the optimal design of interest-rate rules.

The paper is structured as follows. Section 2 outlines the environment. The equilibrium without the money market and the optimal monetary policy are derived in Section 3. The equilibrium with the money market is characterized in Section 4. Finally, in Section 5, we discuss the policy implications that arise from the model, and Section 6 concludes. All proofs and a description of the Euro money markets and the ECB's operating procedures can be found in the Appendix.

\footnotetext{
${ }^{8}$ This fact is also emphasized in Woodford's (2003) book at the beginning of Chapter 2: "While virtually all central banks use short-term nominal interest rates (..) as their instrument (...), the theoretical literature in monetary economics has concerned itself almost entirely with the analysis of policies that are described by alternative (...) paths for the money supply. The aim of this chapter is to remedy this oversight by presenting a theory of price-level determination under interest-rate rules of the sort that are often taken to describe actual central-bank policies."
} 


\section{Environment}

We construct a dynamic general equilibrium model with a [0,1]-continuum of infinitelylived agents and a central bank. ${ }^{9}$ Time is discrete, and in each period three perfectly competitive markets open sequentially. The first market is the settlement market, where all agents produce and consume a general good and settle their claims from the previous period. The second market is the money market, where agents can borrow and lend cash at the market rate, and the third market is a goods market, where agents either produce or consume a perishable good.

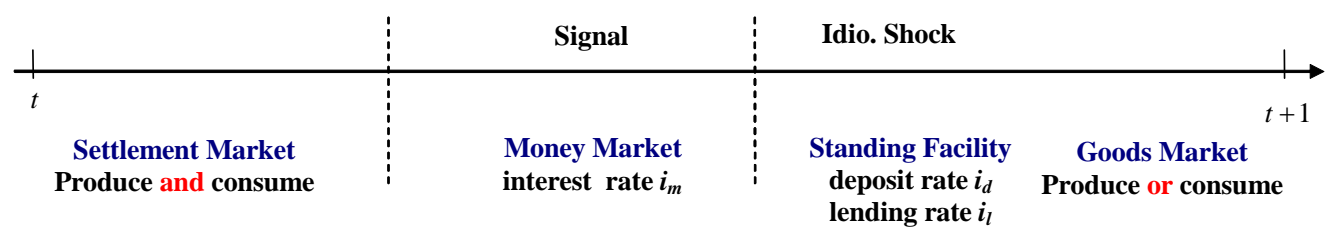

Figure 3: Sequence of markets.

We now describe each market starting from the last one.

Goods market At the beginning of the third market, agents receive idiosyncratic preference and technology shocks which determine whether they consume or produce in this market. With probability $1-n$ an agent can consume and cannot produce: we refer to these agents as buyers. With probability $n$, an agent can produce and cannot consume: these are sellers. Agents get utility $u(q)$ from $q$ consumption in the third market, where $u^{\prime}(q)>0, u^{\prime \prime}(q)<0, u^{\prime}(0)=+\infty$ and $u^{\prime}(\infty)=0$. Producers incur a utility $\operatorname{cost} c(q)=q$ from producing $q$ units of output. All trades are anonymous, and agents' trading histories are private information. Since sellers require immediate

\footnotetext{
${ }^{9}$ The environment combines elements of Lagos and Wright (2005) and Berentsen, Camera and Waller (2006). The Lagos-Wright framework provides a microfoundation for money demand while keeping the distribution of money balances analytically tractable. Berentsen, Camera and Waller (2006) introduce financial intermediation into the Lagos-Wright framework. The sequence of markets is motivated by the ECB's operating procedures. In the Appendix, we describe the functioning of the Euro money markets and the ECB's operating procedures.
} 
compensation for their production effort, money is essential for trade. The discount factor is $\beta$ where for technical reasons we assume that $\beta>n$.

Money market At the beginning of the second market, agents receive a signal about the probability that they will become a consumer or a producer in the goods market. With probability $\sigma^{k}$, an agent receives the information that he will be a seller with probability $n^{k}, k=H, L$, where $\varepsilon \equiv n^{H}-n^{L} \in[0,1]$. We assume that $n=\sum_{k=H, L} \sigma^{k} n^{k}$ so that there is no aggregate uncertainty. This modeling approach captures the idea that, when the money market is open, agents receive information about their end-of-day cash holdings. Some agents believe that they are likely to have excess cash at the end of the day, and others that they are likely to be short of cash. The difference in expected liquidity needs generates an incentive for trading in the money market.

There are three cases. If $\varepsilon=0$, the signal contains no information and so agents have no gains from trading in the money market. ${ }^{10}$ Consequently, no trade occurs in the money market. If $\varepsilon=1$, there is no uncertainty about the liquidity shock in the goods market. Consequently, the portfolios are completely adjusted in the money market and no agent accesses the standing facility. Finally, if $\varepsilon \in(0,1)$, the signal contains some information about the future liquidity shock, but the information is not perfect. As a result, agents use both the money market and the standing facility to adjust their portfolio. For example, some agents will get the information that they will be sellers with high probability but then turn out to be buyers. These agents will first use the money market to trade away their cash and then use the standing facility to take out loans.

Settlement market In the first market, agents produce and consume general goods, repay loans, redeem deposits and adjust their money balances. General goods are produced solely from inputs of labor according to a constant return to scale production technology where one unit of the consumption good is produced with one

\footnotetext{
${ }^{10}$ Here, we already take into account that in equilibrium all agents leave the settlement stage with the same amounts of money and bonds (see the analysis in Section 3.1). Consequently, in equilibrium all agents are identical at the beginning of the money market. Thus, when $\varepsilon=0$ they remain identical and so there are no gains from trading in the money market.
} 
unit of labor generating one unit of disutility. Thus, producing $h$ units of the general good implies disutility $-h$, while consuming $h$ units gives utility $h$. The purpose of this market is that agents can settle their debt. A convenient feature of these assumptions about preferences and technology is that they keep the distribution of money balances analytically tractable as in Lagos and Wright (2005). ${ }^{11}$ As we will see below, in equilibrium all households will hold the same amounts of money and collateral when they move on to the money market. ${ }^{12}$

\subsection{Standing facility}

We assume that at the beginning of the third market, after the idiosyncratic shocks are observed, a central bank offers a borrowing facility and a deposit facility. The central bank operates at zero cost and offers nominal loans $\ell$ at an interest rate $i_{\ell}$ and promises to pay interest rate $i_{d}$ on nominal deposits $d$ with $i_{\ell} \geq i_{d}$. This condition eliminates the possibility for arbitrage where agents borrow and subsequently make a deposit at interest $i_{d}>i_{\ell}$, thus increasing their money holdings at no cost.

Since we focus on standing facilities, we restrict financial contracts to overnight contracts. An agent who borrows $\ell$ units of money from the central bank in market 3 , repays $\left(1+i_{\ell}\right) \ell$ units of money in market 1 of the following period. Also, an agent who deposits $d$ units of money at the central bank in market 3 of period $t$ receives $\left(1+i_{d}\right) d$ units of money in market 1 of the following period.

Collateral In any model of credit, default is a serious issue. Since production is costly, those agents who have borrowed in the previous period have an incentive to default in market 1 of the current period. To prevent default, all loans must be secured with collateral. We assume that general goods produced in market 1 can be stored and used as collateral. The storage technology has constant return to scale and yields $R \geq 1$ units of general goods in market 1 of the following period. We impose $\beta R \leq 1$, since when $\beta R>1$, agents would store infinite amounts of goods which is

\footnotetext{
${ }^{11}$ As in Koeppl, Monnet and Temzelides (2006) the linearity of consumption utility and the linearity of production disutility implies that no welfare is generated.

${ }^{12}$ An alternative framework that would also generate a degenerated distribution of asset holdings is the large household framework of Shi (1997).
} 
inconsistent with equilibrium.

The central bank operates the money market and keeps track of all financial arrangements and collateral holdings. In particular, only the central bank can verify the existence of collateral. This means that collateral cannot be used to secure trade credit between a seller and a buyer in the goods market.

Monetary policy Define $\delta \equiv i_{\ell}-i_{d}$ and note that the central bank can change policy in two ways. It can either increase or decrease $\delta$, holding the policy rate (or target rate $) i_{p}=\left(i_{\ell}+i_{d}\right) / 2$ constant, or it can change $i_{p}$ while holding $\delta$ constant.

In a channel system, the money stock evolves endogenously as follows

$$
M_{+1}=M-i_{\ell} L+i_{d} D+\pi M
$$

where $M$ denotes the per capita stock of money at the beginning of period $t$. In the first market, total loans $L$ are repaid. Since interest-rate payments by the agents are $i_{\ell} L$, the stock of money shrinks by this amount. Interest payments by the central bank on total deposits are $i_{d} D$. The central bank simply prints additional money to make these interest payments so the stock of money increases by this amount. Finally, the central bank can also change the stock of money via lump-sum transfers $T=\pi M$ in market 1 . However, since central banks cannot tax agents, we restrict these lump-sum transfers to be positive, that is $\pi \geq 0 .{ }^{13}$

\section{$2.2 \quad$ First-best allocation}

In the Appendix, we show that the expected lifetime utility of the representative agent for a stationary allocation $(q, b)$, where $q$ is consumption and $b$ collateral holdings at the beginning of a period, is given by

$$
(1-\beta) \mathcal{W}=(1-n)[u(q)-q]+(\beta R-1) b .
$$

The first term on the right-hand side of the equation is the expected utility from consuming and producing the market 3 good. The second term is the utility of

\footnotetext{
${ }^{13}$ The lump-sum transfers are a substitute for open-market operations that we do not model here. However, in pure channel systems central banks do not use open-market operations to affect the money market rate on a regular basis. Nevertheless, there is no clear reason why we should rule this possibility out. Later we will show that it is optimal to set $\pi=0$.
} 
producing collateral and receiving the return in the following period.

It is obvious that the first-best allocation $\left(q^{*}, b^{*}\right)$ satisfies $q=q^{*}$, where $q^{*}$ is the value of $q$ that solves $u^{\prime}(q)=1$. Moreover, $b^{*}=0$ if $\beta R<1$ and $b^{*}$ is indeterminate if $\beta R=1$. Thus, a social planner would never choose a positive amount of collateral when collateral is costly.

\section{No trade in the money market}

In this section, to focus on the stabding facility, we consider the case, where the signal contains no information, i.e., $\varepsilon=0$. In this case, agents have no gains from trading in the money market. Consequently, no trade occurs in the money market, and agents only use the lending and deposit facilities of the central bank to adjust their money holdings. We will consider the case $\varepsilon \in(0,1)$ in Section 4 .

In period $t$, let $\phi \equiv 1 / P$ be the real price of money in market 1 , where $P$ is the price of goods in market 1 . We focus on symmetric and stationary equilibria, where all agents follow identical strategies and where the real allocation is constant over time. In a stationary equilibrium, beginning-of-period real money balances are time-invariant

$$
\phi M=\phi_{+1} M_{+1} .
$$

This implies that $\phi / \phi_{+1}=P_{+1} / P=M_{+1} / M$ is constant. Denote $\gamma \equiv M_{+1} / M$ the time-invariant (endogenous) growth rate of the money supply.

We let $V(m, b)$ denote the expected value from entering market 2 with $m$ units of money and $b$ collateral. $W(m, b, \ell, d)$ denotes the expected value of entering the first market with $m$ units of money, $b$ collateral, $\ell$ loans, and $d$ deposits. For notational simplicity, we suppress the dependence of the value function on the time index $t$.

In what follows, we look at a representative period $t$.

\subsection{Settlement}

In the first market, the problem of a representative agent is:

$$
\begin{gathered}
W(m, b, \ell, d)=\max _{h, m_{2}, b_{2}}-h+V\left(m_{2}, b_{2}\right) \\
\text { s.t. } \quad \phi m_{2}+b_{2}=h+\phi m+R b+\phi\left(1+i_{d}\right) d-\phi\left(1+i_{\ell}\right) \ell+\phi \pi M .
\end{gathered}
$$


where $h$ is hours worked in market $1, m_{2}$ is the amount of money brought in to the second market, and $b_{2}$ is the amount of collateral brought in to the second market. Using the budget constraint to eliminate $h$ in the objective function, one obtains the first-order conditions ${ }^{14}$

$$
\begin{aligned}
& V_{m} \leq \phi(=\text { if } m>0) \\
& V_{b} \leq 1(=\text { if } b>0)
\end{aligned}
$$

$V_{m} \equiv \frac{\partial V\left(m_{2}, b_{2}\right)}{\partial m_{2}}$ is the marginal value of taking an additional unit of money into the second market in period $t$. Since the marginal disutility of working is one, $-\phi$ is the utility cost of acquiring one unit of money in the first market of period $t . V_{b} \equiv \frac{\partial V\left(m_{2}, b_{2}\right)}{\partial b_{2}}$ is the marginal value of taking additional collateral into the second market in period $t$. Since the marginal disutility of working is $1,-1$ is the utility cost of acquiring one unit of collateral in the first market of period $t$. The implication of (4) and (5) is that all agents enter the following period with the same amount of money and the same quantity of collateral (which can be zero). This is the reason why we interpret this market as a settlement stage. By itself, this market does not increase social welfare. Rather, it involves a mere transfer of an asset between participants in order to settle claims from the previous period.

The envelope conditions are

$$
W_{m}=\phi ; W_{b}=R ; W_{\ell}=-\phi\left(1+i_{\ell}\right) ; W_{d}=\phi\left(1+i_{d}\right)
$$

where $W_{j}$ is the partial derivative of $W(m, b, \ell, d)$ with respect to $j=m, b, \ell, d$.

\subsection{Liquidity shocks}

We immediately proceed to market 3 since, when $\varepsilon=0$, no trade occurs in the money market. At the beginning of market 3, agents receive idiosyncratic shocks which determine whether they are consumers or producers. With probability $1-n$, an agent becomes a consumer, and, with probability $n$, a producer. Let $q$ and $q_{s}$ respectively denote the quantities consumed by a buyer and produced by a seller in market 3 . Let $\ell_{b}\left(\ell_{s}\right)$ and $d_{b}\left(d_{s}\right)$ respectively denote the loan obtained and the

\footnotetext{
${ }^{14}$ Throughout the paper, we focus on monetary equilibria, $m>0$, where (4) holds with equality.
} 
amount of money deposited by a buyer (seller) in market 3. An agent who has $m$ money and $b$ collateral at the opening of market 3 has expected lifetime utility

$$
\begin{aligned}
V(m, b)= & (1-n)\left[u(q)+\beta W\left(m-p q-d_{b}+\ell_{b}, b, \ell_{b}, d_{b}\right)\right] \\
& +n\left[-q_{s}+\beta W\left(m+p q_{s}-d_{s}+\ell_{s}, b, \ell_{s}, d_{s}\right)\right]
\end{aligned}
$$

where $q, q_{s}, \ell_{s}, \ell_{b}, d_{s}$ and $d_{b}$ are chosen optimally as follows.

It is obvious that buyers will never deposit funds in the central bank and sellers will never take out loans and therefore $d_{b}=0$ and $\ell_{s}=0$. For the rest of the paper, to simplify notation, let $\ell \equiv \ell_{b}$ and $d \equiv d_{s}$. Accordingly, we get

$$
\begin{aligned}
V(m, b)= & (1-n)[u(q)+\beta W(m-p q+\ell, b, \ell, 0)] \\
& +n\left[-q_{s}+\beta W\left(m+p q_{s}-d, b, 0, d\right)\right]
\end{aligned}
$$

where $q_{s}, q, \ell$ and $d$ solve the following optimization problems.

A seller's problem is $\max _{q_{s}, d}\left[-q_{s}+\beta W\left(m+p q_{s}-d, b, 0, d\right)\right]$ s.t. $m+p q_{s}-d \geq 0 .{ }^{15}$ Using (6), the first-order condition reduces to

$$
\begin{aligned}
p \beta \phi_{+1}+p \beta \phi_{+1} \lambda_{d} & =1 \\
i_{d} & =\lambda_{d}
\end{aligned}
$$

where $\beta \phi_{+1} \lambda_{d}$ is the multiplier on the deposit constraint. The two conditions can be combined to get

$$
p \beta \phi_{+1}\left(1+i_{d}\right)=1 \text {. }
$$

If an agent is a buyer, he solves the following maximization problem:

$$
\begin{array}{cl}
\max _{q, \ell} & u(q)+\beta W(m-p q+\ell, b, \ell, 0) \\
& \text { s.t. } \quad p q \leq m+\ell \text { and } \ell \leq \bar{\ell}
\end{array}
$$

where

$$
\bar{\ell} \equiv R b /\left[\phi_{+1}\left(1+i_{\ell}\right)\right]
$$

\footnotetext{
${ }^{15}$ Here, we assume that sellers can deposit their money holdings at the standing facility, including the proceeds from their latest transaction. This is in line with the institutional details described in the Appendix that banks can access the standing facility of the ECB 30 minutes after the close of the money market. The results are not fundamentally affected when agents can only deposit a fraction or none of their receipts from selling goods.
} 
is the maximal amount that a buyer can borrow from the central bank since $b$ units of collateral transform into $R b$ units of real goods at the beginning of the following period. These goods can be sold for $R b / \phi_{+1}$ units of money. Finally, the collateral must also cover the interest payment.

Using (6), the buyer's first-order conditions can be written as

$$
\begin{aligned}
u^{\prime}(q) & =p \beta \phi_{+1}\left(1+\lambda_{q}\right) \\
\lambda_{q} & =\lambda_{\ell}+i_{\ell}
\end{aligned}
$$

where $\beta \phi_{+1} \lambda_{q}$ is the multiplier of the buyer's budget constraint and $\beta \phi_{+1} \lambda_{\ell}$ the multiplier of the borrowing constraint. Using (9) and combining (11) and (12) yields

$$
u^{\prime}(q)=\frac{1+i_{\ell}+\lambda_{\ell}}{1+i_{d}} .
$$

If the borrowing constraint is not binding, and the central bank sets $i_{\ell}=i_{d}$, trades are efficient. If the borrowing constraint is binding, then $u^{\prime}(q)>1$, which means trades are inefficient even when $i_{\ell}=i_{d}$.

Using the envelope theorem and (11), the marginal value of money in market 3 is

$$
V_{m}=(1-n) u^{\prime}(q) / p+n \beta \phi_{+1}\left(1+i_{d}\right)
$$

The marginal value of money has a straightforward interpretation. An agent with an additional unit of money becomes a buyer with probability $1-n$, in which case he acquires $1 / p$ units of goods yielding additional utility $u^{\prime}(q) / p$. With probability $n$, he becomes a seller, in which case he deposits his money overnight, yielding the nominal return $1+i_{d}$. Note that the standing facility increases the marginal value of money because agents can earn interest on idle cash.

\subsection{Liquidity premium}

Since in equilibrium there is no default, the real return of collateral is $\beta R$. The real return, $\beta R$, is smaller than the marginal value, $V_{b}$, if $\lambda_{\ell}>0$. To see this, use the envelope theorem to derive the marginal value of collateral in the third market

$$
V_{b}=(1-n) \lambda_{\ell} \beta R /\left(1+i_{\ell}\right)+\beta R \text {. }
$$


Thus, the difference between the real return and the marginal value is $(1-n) \lambda_{\ell} \beta R /\left(1+i_{\ell}\right)$. This quantity is positive if collateral relaxes the borrowing constraints of the buyers; i.e., if $\lambda_{\ell}>0$. It is critical for the working of the model that $V_{b}>\beta R$. The reason is that, since $\beta R-1$ is negative, agents are only willing to hold collateral if its liquidity value as expressed by the shadow price $\lambda_{\ell}$ is positive.

To derive the liquidity premium on the collateral, use the first-order conditions (5) and (13) to write (15) as follows:

$$
1-\beta R=(1-n)\left[u^{\prime}(q) \beta R / \Delta-\beta R\right]
$$

where $\Delta \equiv\left(1+i_{\ell}\right) /\left(1+i_{d}\right)$. The term $\beta R / \Delta$ is the price of goods in terms of collateral in market 3. A buyer can use the collateral to borrow $\frac{R}{\phi_{+1}\left(1+i_{\ell}\right)}$ units of money which allows him to acquire $\frac{R}{p \phi_{+1}\left(1+i_{\ell}\right)}=\frac{\beta R\left(1+i_{d}\right)}{1+i_{\ell}}=\beta R / \Delta$ units of goods.

The right-hand side of equation (16) is the collateral's liquidity premium. While collateral costs -1 to produce, its return is $\beta R \leq 1$. Hence, if $\beta R<1$, agents need an incentive to hold collateral. This is provided by making collateral liquid. ${ }^{16}$

\subsection{Symmetric stationary equilibrium}

To define a symmetric stationary equilibrium, use the first-order condition (5) and (16) to get

$$
\frac{1-\beta R}{\beta R} \geq(1-n)\left[u^{\prime}(q) / \Delta-1\right](=\text { if } b>0) .
$$

Then (4), (9), (14), and taking into account that in a stationary equilibrium $M_{+1} / M=$ $\phi / \phi_{+1}=\gamma$, yield

$$
\frac{\gamma-\beta\left(1+i_{d}\right)}{\beta\left(1+i_{d}\right)}=(1-n)\left[u^{\prime}(q)-1\right] .
$$

Also, from (1), since $L=(1-n) \ell$ and $D=n d$, we get

$$
\gamma=1+i_{d}-(1-n)\left(i_{\ell}-i_{d}\right) \frac{z_{\ell}}{z_{m}}+\pi
$$

where $z_{m} \equiv m / p$ and $z_{\ell} \equiv \ell / p$. To derive this equation, we use $d=m+p q_{s}$, market clearing $n q_{s}=(1-n) q$, and we take into account that in symmetric equilibrium all

\footnotetext{
${ }^{16}$ In these type of model, liquidity premia arise naturally. See, for example, Lagos (2005), Lester, Postlewaite and Wright (2006), Telyukova and Wright (2006) or Berentsen and Waller (2006).
} 
agents hold identical amounts of money when they enter market 3. Then, from the budget constraint of the buyer, we have

$$
q=z_{m}+z_{\ell}
$$

Finally, since $\beta R<1$ in any equilibrium where agents hold collateral, it must be the case that the borrowing constraint is binding and so from (9) and (10) we get ${ }^{17}$

$$
z_{\ell}=\beta R b / \Delta
$$

We can use these five equations to define a symmetric stationary equilibrium. They determine the endogenous variables $\left(\gamma, q, z_{\ell}, z_{m}, b\right)$. Note that all other endogenous variables can be derived from these equilibrium values.

Definition 1 A symmetric stationary equilibrium is a policy $\left(i_{d}, i_{\ell}, \pi\right)$ and a timeinvariant list $\left(\gamma, q, z_{\ell}, z_{m}, b\right)$ satisfying (17)-(21) with $z_{\ell} \geq 0$ and $z_{m} \geq 0$.

Let

$$
\tilde{\Delta} \equiv \frac{1-\beta n+\pi /\left(1+i_{d}\right)}{1 / R-n \beta}
$$

Then we have the following:

Proposition 1 For any $\left(i_{d}, i_{\ell}, \pi\right)$ with $i_{\ell} \geq i_{d} \geq 0$, there exists a unique symmetric stationary equilibrium such that

$$
\begin{array}{ll}
z_{\ell}>0 \text { and } z_{m}=0 & \text { if and only if } \Delta=1 \\
z_{\ell}>0 \text { and } z_{m}>0 & \text { if and only if } 1<\Delta<\tilde{\Delta} \\
z_{\ell}=0 \text { and } z_{m}>0 & \text { if and only if } \Delta \geq \tilde{\Delta} .
\end{array}
$$

Several points are worth mentioning. First, the critical element to verify in the proof is under which condition agents acquire collateral. They are willing to borrow at the standing facility if the borrowing rate is not too high; i.e., if $\Delta<\tilde{\Delta}$. Second, the critical value $\tilde{\Delta}$ is increasing in $R$ and $\pi$, and so is $b$. Agents increase their collateral

\footnotetext{
${ }^{17}$ If the borrowing constraint is non-binding $\left(\lambda_{\ell}=0\right)$, equation (15) reduces to $V_{b}=\beta R$, implying from (5) that $b=0$ since we have $\beta R<1$. Consequently, in any equilibrium where agents hold collateral, it must be the case that the constraint is binding $\left(\lambda_{\ell}>0\right)$ and so $\ell=\bar{\ell}=R b /\left[\phi_{+1}(1+i)\right]$, implying $\frac{\partial \ell}{\partial b}=R /\left[\phi_{+1}(1+i)\right]$.
} 
holdings and hence finance a larger share of their consumption by borrowing if $R$ or $\pi$ are increased. Third, if $\Delta=1$, agents are not willing to hold money across periods. They just use collateral to borrow money to finance their consumption. This, however, does not mean that money is not used since it still plays the role of a medium of exchange in market 3 . It only means that agents do not want to hold it across periods.

Given a real allocation $(q(\Delta), b(\Delta))$ any pair $\left(i_{\ell}, i_{d}\right)$ satisfying $\Delta=\frac{1+i_{\ell}}{1+i_{d}}$ is consistent with this allocation. Thus, there are many ways to implement a given policy $\Delta$. The allocations only differ in the rate of inflation. This can be seen from (19) which can be written as follows

$$
\frac{\gamma-\pi}{1+i_{d}}=1-(1-n)(\Delta-1) \frac{z_{\ell}}{z_{m}} .
$$

Since the right-hand side of the equation is a constant for a given $\Delta$, the inflation rate $\gamma-1$ is increasing in $i_{d}$. To keep $\Delta$ constant when increasing $i_{d}$, one needs to increase $i_{\ell}$ accordingly.

\subsection{Optimal policy}

We now derive the optimal policy. The central bank's objective is to maximize the expected lifetime utility of the representative agent. It does so by choosing lump-sum transfers $\pi$, consumption $q$ and collateral holding $b$ to maximize (2) subject to the constraint that its choice is consistent with the allocation given by (17)-(20). Given $\pi$, the policy is implemented by choosing $\Delta$.

Assume first that it is optimal to set $\Delta \geq \tilde{\Delta}$. In this case, no agent is borrowing at the standing facility which implies that $b=0$. Moreover, from (18) and (19) $q$ satisfies

$$
\tilde{q}(\pi)=u^{\prime-1}\left(\frac{1-\beta n+\pi /\left(1+i_{d}\right)}{\beta(1-n)}\right) .
$$

Note that $\tilde{q}$ is independent of $\Delta$ when $\Delta \geq \tilde{\Delta}$ and so any $\Delta \geq \tilde{\Delta}$, implements the same real allocation $(b, q)=(0, \tilde{q})$.

Now consider the largest $q$ that the central bank can implement. From (17) the largest $q$ is attained when $\Delta=1$. It satisfies

$$
\hat{q}=u^{\prime-1}\left[\frac{1 /(\beta R)-n}{1-n}\right] .
$$


Thus, the policy $\Delta=1$ attains the allocation $(b, q)=(\hat{q} /(\beta R), \hat{q})$ since no agent is holding money across the period when $\Delta=1$. Accordingly, the central bank is constrained to choose quantities $q$ such that $\hat{q} \geq q \geq \tilde{q}(\pi)$.

Finally, it can be shown (see the proof of Proposition 1) that when $1 \leq \Delta<\tilde{\Delta}$, $b$ and $q$ solve

$$
\begin{aligned}
\frac{1-\beta R}{\beta R} & =(1-n)\left[u^{\prime}(q) / \Delta-1\right] \\
q & =\beta \operatorname{RbF}(\Delta ; \pi)
\end{aligned}
$$

where

$$
F(\Delta ; \pi)=\frac{1}{\Delta}\left[1+\frac{(1-n)(\Delta-1)}{1+\beta n(\Delta-1)-\Delta / R+\pi /\left(1+i_{d}\right)}\right] .
$$

Thus, the central bank is constrained to choose an allocation that satisfies (23) and (24), and so the central bank's maximization problem is

$$
\begin{array}{cc}
\max _{q, b, \pi} & (1-n)[u(q)-q]+(\beta R-1) b \\
\text { s.t. } \quad q= & \beta R b F\left(\frac{\beta R(1-n) u^{\prime}(q)}{1-n \beta R} ; \pi\right) \\
& \text { and } \hat{q} \geq q \geq \tilde{q}(\pi)
\end{array}
$$

where to derive (25), we use (23) to replace $\Delta$ in $(24)$.

Proposition $2 \pi=0$ is optimal. Also, there exists a critical value $\bar{R}$ such that if $R<\bar{R}$, then the optimal policy is $\Delta \geq \tilde{\Delta}$. Otherwise, the optimal policy is $\Delta \in$ $(1, \tilde{\Delta})$.

The striking result of Proposition 2 is that it is never optimal to set a zero interestrate band $\delta=i_{\ell}-i_{d}$ since $\Delta>1$. The reason is that, for society, the use of collateral is costly, since $\beta R-1$ is negative. The benefit is that it increases consumption above $q=\tilde{q}$. The central bank thus faces a trade-off. It can encourage the use of costly collateral to increase consumption. The optimal policy simply equates the marginal benefit of additional consumption to the marginal cost of holding collateral. It is interesting to note that, in contrast to collateral, the use of fiat money is not costly for society since money can be produced without cost. 
If $R$ is small $(R<\bar{R})$, it is optimal for the central bank to discourage the use of collateral. ${ }^{18}$ It does so by implementing an interest-rate policy that satisfies $\Delta \geq \tilde{\Delta}$. In contrast, if the rate of return is sufficiently high, it sets $\Delta \in(1, \tilde{\Delta})$ so that agents finance some of their consumption through borrowing at the standing facility. An increase in $R$ reduces the optimal $\Delta$. In the limit, as $R \rightarrow 1 / \beta$, the holding of collateral becomes costless. We consider the optimal policy in this limiting case below.

An interesting aspect of Proposition 2 is that it is optimal to set $\pi=0$. To see why, note that $\hat{q}$ is independent of $\pi$, and $\tilde{q}$ is decreasing in $\pi$. Therefore, increasing $\pi$ increases the set of attainable allocations, but only by decreasing the lower bound of the feasible $q$ 's. Then, since $F$ is decreasing in $\pi$, an increase in $\pi$ either increases $b$, decreases $q$ or both. This reduces welfare unambiguously. Hence, it is optimal to set $\pi$ to zero. The intuition is that an increase in $\pi$ is equivalent to an increase in inflation. The inflation tax reduces the sellers willingness to produce for money and so agents agents substitute bonds for money.

These results are intuitive. The optimal monetary policy trades off the cost of holding collateral and the consumption flow from borrowing at the facility. When collateral is costly to hold, the central bank wants to discourage its use. This is achieved by increasing the cost of transforming collateral into money; that is by increasing the interest-rate corridor. By modifying the liquidity properties of collateral, monetary policy affects the portfolio decision of agents and, as a consequence, the real allocation.

Costless collateral Holding collateral is costless when $R=1 / \beta$ since the cost of acquiring one unit is equal to the discounted return $\beta R$. To avoid indeterminacies of the equilibrium allocation, we consider the limiting allocation when the rate of return of the collateral satisfies $R \rightarrow 1 / \beta .^{19}$ In this limiting case, the critical value

\footnotetext{
${ }^{18}$ This is similar as in Lagos and Rocheteau (2004), albeit in a very different context. They construct a model where capital competes with fiat money as a medium of exchange. They show that when the socially efficient stock of capital is low (which is the case when the rate of return is low) a monetary equilibrium exists that dominates the non-monetary one in terms of welfare. So in this case, it would be optimal to discourage the use of capital as a medium of exchange.

${ }^{19}$ We consider the limiting allocation since at $R=1 / \beta$ agents are indifferent to how much collateral they acquire even if they plan not to use it to obtain goods. If $\lambda_{\ell}>0$, agents are strictly better off
} 
is $\tilde{\Delta}=\frac{1-\beta n}{\beta-\beta n}>1$ and Proposition 1 continues to hold. We define the allocation that is attained under the optimal policy as the limiting allocation that is attained when $i_{\ell} \rightarrow i_{d}$. We find the following results.

Proposition 3 With costless collateral, the optimal policy $i_{\ell} \rightarrow i_{d}$ implements the first-best allocation $q^{*}$. The price level approaches infinity.

The proof of the first part is an immediate consequence of equation (17) which implies that $\lim _{\beta R \rightarrow 1} u^{\prime}(q)=\Delta$. Since the first-best allocation requires that $u^{\prime}(q)=1$, the result is established.

To understand why the price level approaches infinity under the optimal policy, note that if $i_{\ell}=i_{d}>0$, then money is strictly dominated in return by collateral. The reason is that the collateral can costlessly be transformed into money and so any consumption level that can be achieved with money can be achieved with collateral at no additional cost. However, the collateral has the intrinsic return $\beta R=1$ while the return on money is $\frac{\beta}{\gamma}<1 .^{20}$ Consequently, the demand for money approaches zero. To encourage agents to hold the stock of money, its price must approach zero. This immediately implies that $p \rightarrow+\infty$, and therefore $z_{m}=M_{+1} / p \rightarrow 0$. Only at the Friedman rule $i_{\ell}=i_{d}=0$ are the returns equal and so agents are indifferent between holding money, collateral or both.

\section{Trade in the money market}

We now assume that $\varepsilon>0$. Recall that at the beginning of the money market, agents receive a signal about the probability that they will become a consumer or a producer in the third market. With probability $\sigma^{k}$, an agent receives the information that he will be a seller with probability $n^{k}, k=H, L$.

We focus on the case where $\varepsilon=n^{H}-n^{L}$ is small. This case captures the situation where agents' liquidity needs in the money market are not too different from each other and not too different from their initial beliefs. In this case, they are reluctant by increasing their collateral holdings up to the amount where $\lambda_{\ell}=0$. However, they are indifferent between any amount of collateral that yields $\lambda_{\ell}=0$. In the limiting allocation attained when $R \rightarrow 1 / \beta$, agents acquire the smallest amount consistent with $\lambda_{\ell}=0$.

${ }^{20}$ This follows from (18) together with $u^{\prime}(q)=\Delta$. 
to pledge all their collateral or to sell all their money holdings in the money market. Consequently, the short-selling constraints in the money market are nonbinding. This essentially means that the money market rate remains strictly within the interestrate corridor, which is consistent with the experience of central banks that operate a channel system (see Figure 1 and 2).

In what follows, we look at a representative period $t$. Also, we assume the central bank does not make lump-sum transfers $(\pi=0)$ since we have shown that this is optimal.

Settlement We let $W(m, b, \ell, d, y)$ denote the expected value of entering the first market with $m$ units of money, $b$ collateral, $\ell$ loans, $d$ deposits, and private credit $y$, where $y>0$ means that the agent has borrowed money in the money market of the previous period. $Z(m, b)$ denotes the expected value from entering the money market with $m$ units of money and $b$ collateral.

In the first market, the problem of a representative agent is:

$$
\begin{gathered}
W(m, b, \ell, d, y)=\max _{h, m_{2}, b_{2}}-h+Z\left(m_{2}, b_{2}\right) \\
\text { s.t. } \phi m_{2}+b_{2}=h+\phi m+R b+\phi\left(1+i_{d}\right) d-\phi\left(1+i_{\ell}\right) \ell-\phi\left(1+i_{m}\right) y
\end{gathered}
$$

where $h$ is hours worked in market 1 . The first-order conditions are

$$
\begin{aligned}
Z_{m} & =\phi \\
Z_{b} & \leq 1(=\text { if } b>0) .
\end{aligned}
$$

$Z_{m} \equiv \frac{\partial Z\left(m_{2}, b_{2}\right)}{\partial m_{2}}$ is the marginal value of taking an additional unit of money and $Z_{b} \equiv \frac{\partial Z\left(m_{2}, b_{2}\right)}{\partial b_{2}}$ is the marginal value of taking additional collateral into the money market in period $t$. The envelope conditions are (6) and

$$
W_{y}=-\phi\left(1+i_{m}\right)
$$

where $W_{y}$ is the partial derivative of $W(m, b, \ell, d, y)$ with respect to $y$.

Money market Let $y^{k}, k=L, H$, be the amount of money acquired in the money market. An agent who has $m$ money and $b$ collateral at the opening of market 2 has 
expected lifetime utility

$$
Z(m, b)=\sum_{k=H, L} \sigma^{k} V^{k}\left(m+y^{k}, b, y^{k}\right)
$$

where $y^{k}$ solves

$$
\max _{y^{k}} V^{k}\left(m+y^{k}, b, y^{k}\right) \text { s.t. } y^{k} \leq R b /\left[\phi_{+1}\left(1+i_{m}\right)\right] \text { and } m+y^{k} \geq 0 .
$$

The first-order conditions are

$$
V_{m}^{k}+V_{y}^{k}-\phi_{+1} \beta \lambda_{m \ell}^{k}+\phi_{+1} \beta \lambda_{m d}^{k}=0
$$

where $\phi_{+1} \beta \lambda_{m \ell}^{k}$ is the multiplier on the borrowing constraint in the money market and $\phi_{+1} \beta \lambda_{m d}^{k}$ is the multiplier on the lending constraint. Note that, since in any equilibrium those agents who are likely to become sellers do not borrow money, and those who are likely to become buyers do not lend money, we have $\lambda_{m \ell}^{H}=0$ and $\lambda_{m d}^{L}=0$ so that from (29)

$$
\begin{gathered}
V_{m}^{H}+V_{y}^{H}+\phi_{+1} \beta \lambda_{m d}^{H}=0 \\
V_{m}^{L}+V_{y}^{L}-\phi_{+1} \beta \lambda_{m \ell}^{L}=0 .
\end{gathered}
$$

The marginal value of collateral is $Z_{b}(m, b)=\sum_{k=H, L} \sigma^{k}\left[V_{b}^{k}+\sigma^{k} \beta R \lambda_{m \ell}^{k} /\left(1+i_{m}\right)\right]$. Then (31) gives us

$$
Z_{b}(m, b)=\sum_{k=H, L} \sigma^{k} V_{b}^{k}+\sigma^{L} \beta R \frac{\left(V_{m}^{L}+V_{y}^{L}\right)}{\beta \phi_{+1}\left(1+i_{m}\right)}
$$

since in any equilibrium $\lambda_{m d}^{L}=\lambda_{m \ell}^{H}=0$.

The marginal value of money is $Z_{m}(m, b)=\sum_{k=H, L} \sigma^{k}\left(V_{m}^{k}+\sigma^{k} \beta \phi_{+1} \lambda_{m d}^{k}\right)$. Then from (30) we have

$$
Z_{m}(m, b)=\sigma^{L} V_{m}^{L}-\sigma^{H} V_{y}^{H}
$$

Thus, the marginal value of money at the beginning of the money market is equal to the expected value of using the money to buy goods in market $3, \sigma^{L} V_{m}^{L}$, plus the expected value of lending it in the money market, $-\sigma^{H} V_{y}^{H}$.

Finally, the market clearing condition is

$$
\sum_{k=H, L} \sigma^{k} y^{k}=0
$$


Goods market At the beginning of market 3, an agent's state is revealed. Consider an agent of type $k$ who received the signal that he will be a buyer with probability $1-n^{k}$ and a producer with probability $n^{k}$. Let $q^{k}$ and $q_{s}^{k}$ respectively denote the quantities consumed as a consumer and produced as a producer in market 3 . Let $\ell_{b}^{k}$ $\left(\ell_{s}^{k}\right)$ and $d_{b}^{k}\left(d_{s}^{k}\right)$ respectively denote the loan obtained from the central bank and the amount of money deposited at the central bank by this agent in this market. If this agent holds $m$ money, $b$ collateral and private debt $y$ at the opening of this market, he has expected lifetime utility

$$
\begin{aligned}
V^{k}(m, b, y)= & \left(1-n^{k}\right)\left[u\left(q^{k}\right)+\beta W\left(m-p q^{k}+\ell^{k}, b, \ell^{k}, 0, y\right)\right] \\
& +n^{k}\left[-q_{s}^{k}+\beta W\left(m+p q_{s}^{k}-d^{k}, b, 0, d^{k}, y\right)\right]
\end{aligned}
$$

where $q^{k}, q_{s}^{k}, \ell_{s}^{k}, \ell_{b}^{k}, d_{s}^{k}$ and $d_{b}^{k}$ are chosen optimally as described in Section 3. The only difference is that the constraints in the goods market now take into account an agent's borrowing or lending $y^{k}$ in the money market as follows

$$
\begin{aligned}
\ell^{k} & \leq \bar{\ell}^{k} \equiv R b /\left[\phi_{+1}\left(1+i_{\ell}\right)\right]-y^{k} / \hat{\Delta} \\
p q^{k} & \leq m+\ell^{k} \\
d^{k} & \leq m
\end{aligned}
$$

where $\hat{\Delta} \equiv \frac{1+i_{\ell}}{1+i_{m}}$. The quantity $\bar{\ell}^{k}$ is still the maximal amount that a buyer can borrow from the central bank. If the agent has borrowed money in the money market $\left(y^{k}>0\right)$, the maximal loan size is reduced by $y^{k} / \hat{\Delta}$. In contrast, if the agent has lent money $\left(y^{k}<0\right)$, it is increased accordingly.

Endogenous money supply Finally, we need to adjust equation (1) to take into account how the money market affects the evolution of the stock of money across periods. The new equation is

$$
M_{+1}=M-\left[\sigma^{H}\left(1-n^{H}\right) \ell^{H}+\sigma^{L}\left(1-n^{L}\right) \ell^{L}\right] i_{\ell}+\left[\sigma^{H} n^{H} d^{H}+\sigma^{L} n^{L} d^{L}\right] i_{d}
$$

where $\ell^{k}=R b /\left[\phi_{+1}\left(1+i_{\ell}\right)\right]-y^{k} / \hat{\Delta}$ and $d^{k}=M+y^{k}+p q_{s}^{k}$. Using the market clearing conditions in the goods and money markets, we can write this equation as follows

$$
M_{+1} / M=1+i_{d}-\left(i_{\ell}-i_{d}\right)\left[\sigma^{L}\left(1-n^{L}\right) \ell^{L} / M+\sigma^{H}\left(1-n^{H}\right) \ell^{H} / M\right] .
$$


It is interesting to compare (39) with (19) (when $\pi=0$ ). As before, the entire stock of money earns interest $i_{d}$. The only difference is the amount of loans that the central bank provides. Without the money market, the amount is $(1-n) \ell / M$; with a money market, it is $\sigma^{L}\left(1-n^{L}\right) \ell^{L} / M+\sigma^{H}\left(1-n^{H}\right) \ell^{H} / M$.

\subsection{Symmetric stationary equilibrium}

We again focus on symmetric and stationary equilibria, where all agents follow identical strategies and where the real allocation is constant over time. Furthermore, we focus on equilibria, where the short-selling constraints in the money market are nonbinding. This essentially means that the money market rate remains strictly within the interest-rate corridor, which is consistent with the experience of central banks that operate a channel system (see Figure 1 and 2).

In the Appendix we prove:

Lemma 4 A symmetric stationary equilibrium where no short-selling constraint is binding in the money market is a time-invariant list $\left(\hat{\Delta}, q^{L}, q^{H}, z^{L}, z^{H}, z_{m}, b, \gamma\right)$ and a policy $\left(i_{d}, i_{\ell}\right)$ satisfying

$$
\begin{aligned}
R \gamma & =1+i_{m} \\
\frac{\beta R b}{\Delta} & =\sigma^{H} q^{H}+\sigma^{L} q^{L}-z_{m} \\
z^{H} & =-\sigma^{L}\left(q^{L}-q^{H}\right)\left(\frac{\hat{\Delta}}{\hat{\Delta}-1}\right)=-\frac{\sigma^{L}}{\sigma^{H}} z^{L} \\
z_{m} & =\left(\frac{\hat{\Delta}}{\hat{\Delta}-1}\right) \frac{\left\{(\hat{\Delta}-1)\left[\sigma^{L}\left(1-n^{L}\right) q^{L}+\sigma^{H}\left(1-n^{H}\right) q^{H}\right]-\varepsilon \sigma^{L} \sigma^{H}\left(q^{L}-q^{H}\right) \hat{\Delta}\right\} R(\Delta-1)}{R \hat{\Delta}-\Delta+(1-n) R \hat{\Delta}(\Delta-1)} \\
\hat{\Delta} & =\frac{\Delta}{n \beta R(1-\Delta)+\Delta} \\
u^{\prime}\left(q^{k}\right) & =\frac{n^{k}}{1-n^{k}} \Delta \frac{1-n \beta R}{n \beta R}, \quad k=H, L,
\end{aligned}
$$

with $b \geq 0, z^{L}<\beta R b \hat{\Delta} / \Delta$ and $z^{H}>-z_{m}$.

We discuss the policy implications of Lemma 4 in Section 5.

Proposition 5 For any $1<\Delta<\tilde{\Delta}$, there exists a critical value $\varepsilon_{1}>0$, defined in the proof, such that, if $\varepsilon<\varepsilon_{1}$, a symmetric monetary equilibrium exists where no short-selling constraint in the money market binds. 
Note first that the system of equations (40) - (45) can be solved recursively. Equations (44) and (45) yield $\hat{\Delta}, q^{L}$ and $q^{H}$. Using these values, we can then derive $\left(z^{L}, z^{H}, z_{m}, b, \gamma\right)$ from the remaining equations. One then has to check that the required inequalities hold. The inequality $b \geq 0$ simply requires that policy is such that agents have an incentive to acquire collateral which is satisfied whenever $\Delta<\tilde{\Delta}$ (defined by (22)). The inequality $z^{L}<\beta R b \hat{\Delta} / \Delta$ requires that those agents who are likely to become buyers are not pledging all their collateral to acquire money in the money market, and the inequality $z^{H}>-z_{m}$ requires that those agents who are likely to become sellers are not selling all their money.

\section{Discussion of the policy implications}

We now discuss the key implications of our model for the behavior of the money market rate, inflation, liquidity, collateral requirement, and the use of interest-rate rules to study monetary policy. These results can be found by inspecting equations (40) and (44). For this discussion, let us define the policy interest rate $i_{p} \equiv\left(i_{\ell}+i_{d}\right) / 2$.

Money market rate In the introduction, we have seen that the money market rate tends to be in the middle or above the target rate, and changes one-to-one with a shift in the corridor (see also Figures 1 and 2). Our model replicates these facts. To see this, we can write (44) as follows

$$
i_{m}=i_{\ell}-n \beta R \delta
$$

where $\delta=i_{\ell}-i_{d}$. Inspection of (46) reveals the following result: First, if the spread $\delta$ is kept constant, $i_{m}$ changes in $i_{\ell}$ one-to-one. Second, if $n \beta R=1 / 2$, then $i_{m}=i_{p}{ }^{21}$ Our model suggests that the money market interest rate lies exactly on the policy rate if, for example, $n=1 / 2$ and $\beta R \rightarrow 1$. It is reasonable to assume that $n=1 / 2$ since it means that on average a bank is equally likely to borrow or to provide cash in the money market and also equally likely to be either short of money or have excess cash at the end of the day. The second assumption means that holding collateral has

\footnotetext{
${ }^{21}$ The first two results exactly match the behavior of the overnight money market rate of the channel system operated by the Reserve Bank of New Zealand. This can be seen from Figure 2 in the introduction.
} 
no cost. Third, as mentioned in the introduction, the Euro money market rate tends to be above the minimum bid rate $i_{p}{ }^{22}$ Our model has a simple explanation for this observation. With $n=1 / 2$ and $\beta R<1$, we have $i_{m}=\frac{i_{\ell}(2-\beta R)+i_{d} \beta R}{2}>i_{p}$. Thus, costly collateral generates a money market rate that tends to be above the target rate.

Inflation To see the implications of our model for inflation, $\pi \equiv \gamma-1,{ }^{23}$ we can rewrite (40) as follows

$$
\pi=\left(1+i_{m}\right) / R-1
$$

By defining $r \equiv R-1$, we get the standard expression for the Fisher equation $(1+r)(1+\pi)=1+i_{m}$. It is interesting to note that the nominal interest rate of the Fisher equation is the money market rate $i_{m}$ and not $i_{\ell}$ or $i_{d}$. Using (46), we can rewrite the Fisher equation as follows:

$$
\pi=\left(1+i_{\ell}\right) / R-n \beta \delta-1
$$

From this expression, it is clear that inflation is increasing in $i_{\ell}$ and $i_{d}$. If we keep the spread $\delta$ constant, and shift the corridor up, inflation is also increasing. Finally, inflation is also increasing if we increase the spread $\delta$ symmetrically around the policy rate when $n<1 /(2 \beta R)$. As we have argued above, this condition is likely to be fulfilled since on average $n=1 / 2$, which implies that the inequality reduces to $\beta R<1$.

Liquidity We can interpret $n$ as a measure for liquidity in the money market. If $n=1 / 2$, as mentioned above, banks are equally likely to have excess money or too little money at the end of the day. If $n<1 / 2$, a bank is more likely to be short of money at the end of the day. The implications of changes in $n$ for the money market rate, can again be explored by considering (44). From this equation it is clear that an increase in liquidity (i.e., an increase in $n$ ) reduces the money market rate. Furthermore, the choice of $n$ affects how close the money market rate is to the policy rate.

\footnotetext{
${ }^{22}$ The European Central Bank does not consider the minimum bid rate to be its target rate. Nevertheless, the minimum bid rate is in the middle of the corridor and, therefore, equal to $i_{p}=\frac{i_{\ell}+i_{d}}{2}$.

${ }^{23}$ Since we study steady state allocations, money growth and inflation are perfectly correlated. Then, through the Fisher equation, long-run money growth and interest rates are positively correlated, as confirmed by the data (see McCandless and Weber 1995).
} 
Collateral requirement In the introduction, we have asked the question what the optimal collateral requirement is? Inspection of (46) reveals that a higher return on collateral, $R$, reduces the money market rate. From (47), one can also see that an increase in $R$, reduces inflation, and, as discussed above, gets the money market rate closer to the target rate. But the most important aspect of the collateral requirement is that it affects the real allocation. Inspection of (45) reveals that an increase in $R$ yields higher consumption, and, consequently, higher welfare.

Interest-rate rules Finally, a central bank can tighten its policy without changing its policy rate by simply increasing the corridor symmetrically around the policy rate. This can be seen by rewriting $\Delta$ as follows

$$
\Delta=\frac{1+i_{\ell}}{1+i_{d}}=\frac{1+i_{\ell}}{2 i_{p}-i_{\ell}}
$$

It is evident that $\partial \Delta / \partial i_{\ell}>0$. Hence, from (45), a symmetric increase of the spread around the policy rate decreases consumption.

There is an important implication from this result. In a channel system, interestrate rules (i.e., rules that specify a path for the policy rate $i_{p}$ ) are meaningless. The reason is that such a rule does not determine whether a policy is "tight" or "loose." Rather, in a channel system, any policy must be characterized through an interest-rate corridor rule.

\section{Conclusion}

We have analyzed the theoretical properties of a channel system of interest-rate control in a dynamic general equilibrium model with infinitely-lived agents and a central bank. With this model, we could match several stylized facts regarding the use of channel systems by central banks. Moreover, we could derive several policy implications that we have summarized in Section 5. Perhaps, the most important result is that interest-rate rules are meaningless in a channel system. In a channel system, any policy must be characterized through an interest-rate corridor rule. This is a new insight, which goes beyond what we already know from the large and growing body of literature on the optimal design of interest-rate rules. 
While our paper is a first step towards analyzing monetary policy implementation in a channel system, many aspects have remained unexplored. Moreover, many elements of real life channel systems are still not very well understood. An indication for this is that many central banks are still experimenting with the optimal design. A point in case is the channel system operated by the Bank of England, that we discuss in the Appendix. One puzzle, for example, is why there is so little volatility in the money-market interest rate in case of New Zealand (see Figure 2) and so much in the cases of England (see Figure 4) and the European Central Bank (Figure 1). What is the role of reserve requirements and should the central bank pay interest on them? Furthermore, we know little about optimal monetary policy in a channel system under stress and aggregate shocks. These are issues left for future research. 


\section{APPENDIX}

\subsection{Background ${ }^{24}$}

To understand some of the features of our environment, it is useful to have some information on how the money market functions and on monetary policy procedures at central banks that operate a standing facility. This section does not aim at being general, and we will, therefore, concentrate on the case of the euro money markets and the ECB's operating procedures.

Operating procedures of the ECB The ECB has two main instruments for the implementation of its monetary policy. First, it conducts weekly main refinancing operations that are collateralized loans with a one-week maturity. Main refinancing operations are implemented using a liquidity auction, where banks bid for liquidity. Bids consist of an amount of liquidity and an interest rate. The total amount to be allocated is announced before the auction. Following the auction, the ECB allocates liquidity according to the bidded rates, in a descending order. The minimum bid rate is the main policy rate used by the ECB to implement monetary policy.

Second, the ECB offers a standing facility with a lending rate that is 100 basis points higher than its minimum bid rate, and a deposit rate that is 100 basis points below its minimum bid rate. At the lending facility, liquidity is provided either in the form of overnight repurchase agreements or as overnight collateralized loans, whereby the ownership of the asset is retained by the debtor. In both cases, banks have to resort to safe, eligible assets as defined by the ECB. Eligible banks can access the standing facilities at any time of the day. The use of the standing facility largely depends on banks' activities on the euro money markets during the day.

The euro money markets There are two segments for the euro money market. The first segment is the unsecured money market, where banks borrow and lend cash to each other without resorting to collateral. The reference interest rate on the unsecured money market is the Euro OverNight Index Average (EONIA), calculated

\footnotetext{
${ }^{24}$ This section draws heavily on materials from ECB (2005), ECB (2004), BIS (2003) and Hartmann, Manna and Manzanares (2001).
} 
by the ECB. The second segment is the secured money market where agents lend at different maturities against collateral. This is the largest money market segment. There are several reference interest rates, depending on maturities (Euro Interbank Offered Rates, or Euribors) and on whether the collateral pledged belong to a general collateral pool (Euripo).

Transactions on both segments of the money market are settled using the two large-value payment systems operating in the euro area, the Trans-European Automated Real-time Gross settlement Express Transfer system (TARGET) and Euro1. These large value payment systems are essential in finalizing the transfer of funds for transactions taking place in money markets. Therefore, the opening and closing hours of money markets are closely related to the operating hours of these payment systems.

TARGET settles payments with immediate finality in central bank money and operates between 7 a.m. and 6 p.m. C.E.T. with a cut-off time of 5 p.m. for customer payments. ${ }^{25}$ Eligible institutions hold accounts at TARGET, which are debited or credited depending on market participants' orders. Intraday credit is provided free of charge as long as it is fully collateralized. Banks may also access the deposit or lending facilities after making a request at the latest 30 minutes after the actual closing time of TARGET. ${ }^{26}$ After the close of TARGET, an overdraft position on a bank's TARGET account is automatically transformed into an overnight loan via a recourse to the lending facility, again against eligible assets.

Euro1 is a private, large-value payment systems offered by the Euro Banking Association (EBA). Euro1 functions as a sort of netting system, whereby on each settlement day, at any given time, each participant will have only one single payment obligation or claim with respect to the community of other participants as joint creditors/debtors. In particular, there are no bilateral payments, claims or obligations between participants. Euro1 settles in central bank money at the ECB at the end of the day. After the cut-off time of 4 p.m. C.E.T., clearing banks with debit positions will pay their single obligations into the EBA settlement account at the ECB through TARGET. After all amounts due have been received, the ECB will pay the clearing

\footnotetext{
${ }^{25}$ The unsecured segment opens around 8 a.m. in the morning and closes around 5:45 p.m.

${ }^{26}$ On the last Eurosystem business day of a minimum reserve maintenance period, the deposit facility can be accessed for 60 minutes after the actual closing of TARGET.
} 
banks with credit positions also using TARGET.

In this paper, we will model two specific features of the description above. First, banks cannot carry overnight overdrafts on their TARGET accounts, and they have to borrow either on the money markets or at the lending facility in order to cover their TARGET positions. When TARGET closes, euro money markets are also closed. As a consequence, the central bank standing facility is, at the end of the day, the only recourse to overnight liquidity. Also, since participants can access the standing facility 30 minutes after the close of target, any late payments received on a TARGET account can be deposited at the standing facility of the ECB. In the first part of the paper, we model this aspect of the liquidity management problem. Second, banks can predict when a payment is due or incoming so that, with a well-functioning money market, the likelihood to resort to the standing facilities should be small. However, there may be unexpected payments to be made that can force banks to hold an overdraft on their TARGET account. In the second part of the paper, we adjunct a money market to the model. There, banks will be able to trade their liquidity when they are confident that they will end up the day with a credit on their central bank account. Given it is the most important segment of the money market, we concentrate on the secured interbank money market.

\subsection{Channel system of the Bank of England}

Here, we discuss the channel system operated by the Bank of England. As shown in Figure 4, the Sterling Overnight Interbank Average rate (SONIA) was very volatile until the first quarter of 2006. Before this date the Bank's implementation framework consisted of a 100-basis-point corridor, non-remunerated daily reserves requirements and a somewhat restricted access to the borrowing facility ${ }^{27}$ From January 2000 to May 2006, the SONIA was on average 5 basis points below the Bank of England target rate, while the daily gilt repo rate with two-week maturity was on average 11 basis points below the target rate over the same period. Furthermore, the Bank decreased its target rate from $4 \%$ to $3.75 \%$ in February 2003. However, the SONIA averaged $3.95 \%$ over the period when the Bank rate was $4 \%$, and averaged $3.76 \%$

\footnotetext{
${ }^{27}$ For details on the Bank of England implementation framework, we refer the reader to Bank of England (2004) and Clews (2005).
} 
after its easing. Hence, while monetary policy targeted a 25-basis-points easing, the Bank effectively implemented a 19-basis-points easing.

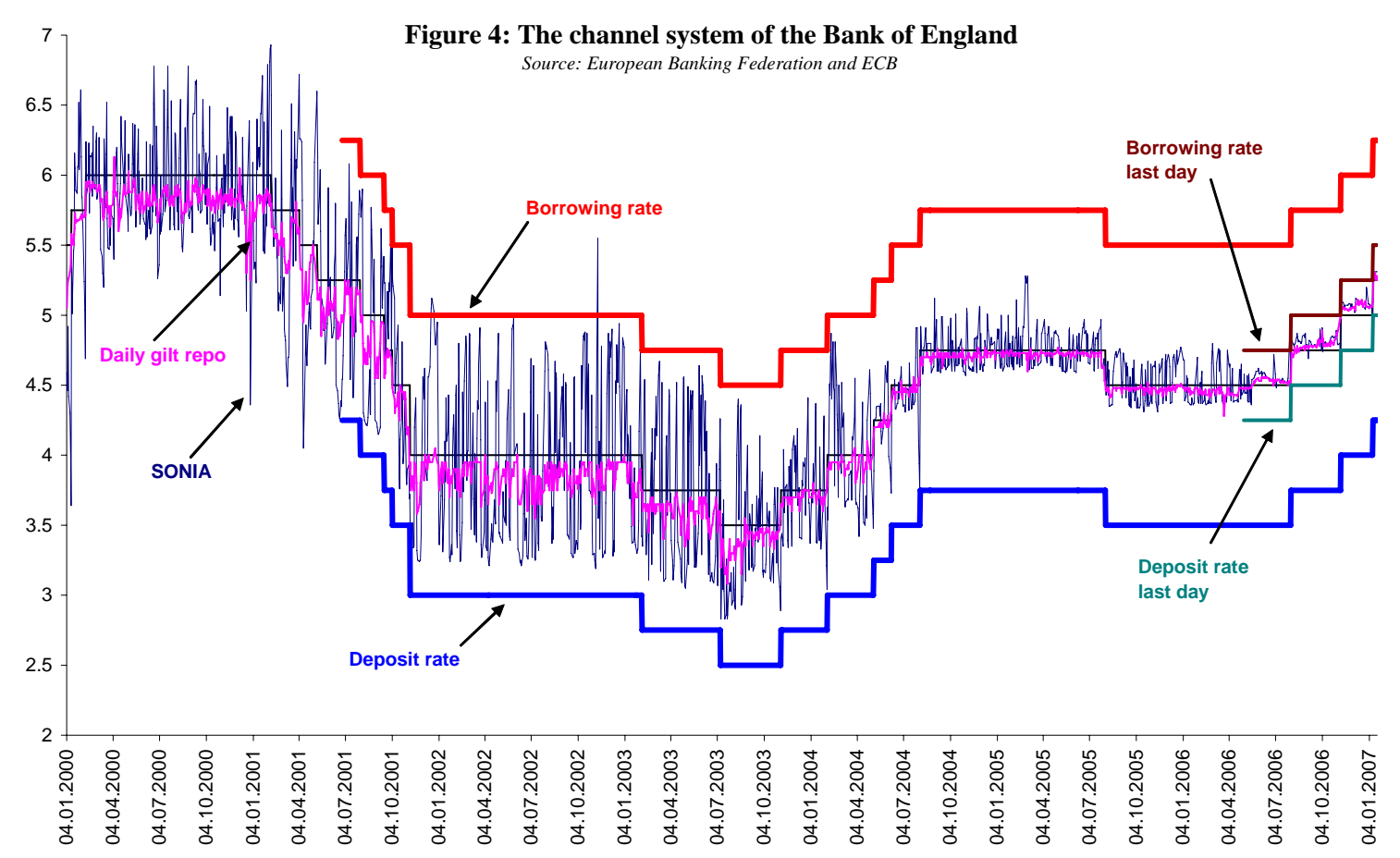

Hence, the implementation framework was not very efficient in implementing monetary policy. As a result of this inefficiency, the Bank of England reformed its implementation framework in 2006. It introduced 1) a 25-basis-point corridor on the last day of the maintenance period, 2) remuneration on reserves within limits at the official bank rate and 3) open market operations to ensure that there is an equal (and small) chance of using either facility. As Figure 4 illustrates, this reform resulted in an immediate decrease in the variability of the SONIA and repo rates. Furthermore, the SONIA is now on average 5 basis points above the Bank's target rate and more surprisingly, the repo rate is also on average 5 basis points above the target rate. Therefore, the reform of the monetary implementation framework increased the average difference between the Bank's target rate and the SONIA by 10 basis points and the difference between the repo rate and the Bank's target rate by 16 basis points. 


\subsection{Welfare}

In this Appendix, we show that, if the central bank's objective is to maximize the expected discounted utility of the representative agent, the central bank's objective is to maximize (2). To derive (2), we must first calculate hours worked in market 1. The money holdings at the opening of the first market are $\tilde{m}=0$, having bought, and $\tilde{m}=m+p q_{s}$, having sold. Hence, hours worked are

$$
\begin{aligned}
& h_{b}=\phi\left[m_{+1}+\left(1+i_{\ell}\right) \ell\right]-(R-1) b-\pi M \\
& h_{s}=\phi\left[m_{+1}-\left(1+i_{d}\right)\left(m+p q_{s}\right)\right]-(R-1) b-\pi M .
\end{aligned}
$$

Since $h=n h_{s}+(1-n) h_{b}$, by using (1) and rearranging we get

$$
\begin{aligned}
h & =-(R-1) b+(1-n) \phi \ell-n \phi\left(m+p q_{s}\right)+\phi m \\
& =-(R-1) b+(1-n) \phi \ell+(1-n) \phi m-n \phi p q_{s} \\
& =-(R-1) b
\end{aligned}
$$

since $p q=m+\ell$ and $q_{s}=\frac{1-n}{n} q$. Then, welfare is given by

$$
\begin{aligned}
\mathcal{W} & =-b+(1-n)[u(q)-q]+\sum_{j=1}^{\infty} \beta^{j}\{(1-n)[u(q)-q]+(R-1) b\} \\
& =\frac{(1-n)[u(q)-q]+(\beta R-1) b}{1-\beta} .
\end{aligned}
$$

\subsection{Proofs}

Proof of Proposition 1. For ease of exposition, we assume $\pi=0$. The proof can be easily replicated when $\pi>0$. We first prove the only if part. Assume first $z_{\ell}=0$ and $z_{m}>0$. Then from (18) and (19) we get

$$
\frac{1-\beta}{\beta}=(1-n)\left[u^{\prime}(q)-1\right]
$$

and from (17) we have

$$
\frac{1-R \beta}{R \beta} \geq(1-n)\left[u^{\prime}(q) / \Delta-1\right] .
$$

Use (49) to replace $u^{\prime}(q)$ in (48) and rearrange to get $\Delta \geq \tilde{\Delta}$. 
Assume now that $z_{\ell}>0$ and $z_{m}>0$. Then from (19) $z_{\ell}>0$ implies that $1+i_{d}>\gamma$. Use (18) to replace $\gamma$ and rearrange to get $\Delta<\tilde{\Delta}$. Next divide (19) by $1+i_{d}$ and solve for $\Delta$ to get

$$
\Delta=1+\frac{z_{m}}{z_{\ell}} \frac{1+i_{d}-\gamma}{(1-n)\left(1+i_{d}\right)}>1
$$

since $1+i_{d}>\gamma$. Hence, we have $1<\Delta<\tilde{\Delta}$, if $z_{\ell}>0$ and $z_{m}>0$.

Finally, assume that $z_{\ell}>0$ and $z_{m}=0$. Then, the previous equation immediately implies that $\Delta=1$.

We now prove the if part. From (18) and (19) we get

$$
1-n \beta-\beta(1-n) u^{\prime}(q)=(1-n)(\Delta-1) \frac{z_{\ell}}{z_{m}}
$$

and from (17) we have

$$
\Delta\left(\frac{1}{R}-n \beta\right) \geq \beta(1-n) u^{\prime}(q)
$$

Assume first that $1<\Delta<\tilde{\Delta}$. Use (50) to rewrite (51) as follows

$$
1-n \beta-\Delta\left(\frac{1}{R}-n \beta\right) \leq(1-n)(\Delta-1) \frac{z_{\ell}}{z_{m}} .
$$

Rearrange to get

$$
0<\tilde{\Delta}-\Delta \leq \frac{(1-n)(\Delta-1)}{(1 / R-n \beta)} \frac{z_{\ell}}{z_{m}} .
$$

Hence, $1<\Delta<\tilde{\Delta}$ implies $\frac{z_{\ell}}{z_{m}}>0$.

Assume next that $\Delta \geq \tilde{\Delta}$. Then from (50) we have

$$
1-n \beta-\beta(1-n) u^{\prime}(q) \geq(1-n)(\tilde{\Delta}-1) \frac{z_{\ell}}{z_{m}} .
$$

Then $z_{\ell}>0$ immediately implies that

$$
0>\tilde{\Delta}-\Delta \geq \frac{(1-n)(\tilde{\Delta}-1)}{(1 / R-n \beta)} \frac{z_{\ell}}{z_{m}}
$$

which is a contradiction. Hence $\Delta \geq \tilde{\Delta}$ implies $z_{\ell}=0$.

Existence and uniqueness when $\tilde{\Delta} \leq \Delta$ : In this case $z_{\ell}=b=0$ and from (19) $\gamma=1+i_{d}$. Then, from (18) and (19) we get (48). Since the right-hand side of (48) is strictly decreasing in $q$, there exists a unique $q$ that solves (48). Finally, from (20) we have $z_{m}=q$. 
Existence and uniqueness when $1<\Delta<\tilde{\Delta}$ : The system of equations (17)(20) with $z_{\ell}=\beta R b / \Delta$ can be reduced as follows. Equations (20) and $z_{\ell}=\beta R b / \Delta$ imply $z_{m}=q-\beta R b / \Delta$. Then, multiply both sides of (19) by $z_{m}$ and replace $z_{m}$ to get

$$
(q-\beta R b / \Delta)\left[\gamma-\left(1+i_{d}\right)\right]=-(1-n) z_{\ell}\left(i_{\ell}-i_{d}\right)
$$

Use (18) to eliminate $\gamma$ and rearrange to get

$$
(q-\beta R b / \Delta)\left\{1-(1-n) \beta\left[u^{\prime}(q)-1\right]-\beta\right\}=(1-n) \frac{\beta R b}{\left(1+i_{\ell}\right)}\left(i_{\ell}-i_{d}\right) .
$$

Hence, an equilibrium is defined by the following two equations:

$$
\begin{aligned}
& \frac{1}{R \beta}=(1-n) u^{\prime}(q) / \Delta+n \\
& (q-\beta R b / \Delta)\left\{1-(1-n) \beta\left[u^{\prime}(q)-1\right]-\beta\right\}=(1-n) \frac{\beta R b}{\left(1+i_{\ell}\right)}\left(i_{\ell}-i_{d}\right)
\end{aligned}
$$

We can use the first equation to replace for $u^{\prime}(q)$ in the second to get

$$
\begin{aligned}
& \frac{1}{R \beta}=(1-n) u^{\prime}(q) / \Delta+n \\
& q=\beta R b F(\Delta) .
\end{aligned}
$$

If we substitute $q$ in the first expression, we get

$$
\frac{1}{R \beta}=(1-n) u^{\prime}[\beta R b F(\Delta)] / \Delta+n \equiv R H S .
$$

The left-hand side of (52) is constant while the right-hand side is decreasing in $b$ for a given $1 \leq \Delta<\tilde{\Delta}$. Moreover, we have $\lim _{b \rightarrow 0} R H S=+\infty$ and $\lim _{b \rightarrow \infty} R H S=n<$ $\frac{1}{R \beta}$. Hence, for any policy $\Delta$ with $1 \leq \Delta<\tilde{\Delta}$, a unique $b>0$ exists. Then, from (24) a unique value for $q$ exists. Accordingly, a unique symmetric stationary equilibrium exists.

Finally, we have $\lim _{\Delta \rightarrow \tilde{\Delta}} F(\Delta)=+\infty$ and so $b \rightarrow 0$.

Proof of Proposition 2. We first show that $\pi=0$ is optimal. Note that $\hat{q}$ is independent of $\pi$, and $\tilde{q}$ is decreasing in $\pi$. Therefore, increasing $\pi$ does only decrease the lower bound of the set of attainable allocations. Second, $F(\Delta ; \pi)$ is decreasing in $\pi$, so that an increase in $\pi$ either increases $b$, decrease $q$, or both. This reduces welfare. Hence, since $\pi \geq 0$, it is optimal to set $\pi$ to zero. 
We now assume $\pi=0$. Substituting (25) into the objective function, the problem becomes

$$
\max _{q}(1-n)[u(q)-q]+(\beta R-1) \frac{q}{\beta R F\left(\frac{R \beta(1-n) u^{\prime}(q)}{1-n R \beta}\right)}
$$

s.t. $\hat{q} \geq q \geq \tilde{q}$.

After rearranging, the first-order condition is

$$
(1-n)\left[u^{\prime}(q)-1\right]+\frac{1-\beta R}{\beta R F(\Delta)}\left[\frac{F^{\prime}(\Delta) \Delta}{F(\Delta)} \frac{u^{\prime \prime}(q) q}{u^{\prime}(q)}-1\right]=\hat{\lambda}-\tilde{\lambda}
$$

where $\hat{\lambda}$ is the multiplier of the first inequality, and $\tilde{\lambda}$ is the multiplier of the second inequality. Consider the first-order condition and note that

$$
\Delta(q)=\frac{R \beta(1-n) u^{\prime}(q)}{1-n R \beta} .
$$

Suppose that the optimal $q$ is such that $\Delta=1$; i.e., $q=\hat{q}$. In this case, $\tilde{\lambda}=0$ and $\hat{\lambda}>0$, implying that $\Theta(\hat{q}, R)>0$. Then we have $F(1)=1, F^{\prime}(1)=\frac{1-n R}{R-1}$ and so

$$
\Theta(\hat{q}, R)=\frac{1-\beta R}{\beta R} \frac{1-n R}{R-1} \frac{u^{\prime \prime}(\hat{q}) \hat{q}}{u^{\prime}(\hat{q})}<0
$$

which is a contradiction. Thus, in any equilibrium $q<\hat{q}$, implying $\Delta>1$.

Now suppose that the optimal $q$ is such that $\Delta=\tilde{\Delta}$; i.e., $q=\tilde{q}$. In this case, $\tilde{\lambda}>0$ and $\hat{\lambda}=0$, implying that $\Theta(\tilde{q}, R)<0$. One can show that $\lim _{\Delta \rightarrow \tilde{\Delta}} F(\Delta)=\infty$, $\lim _{\Delta \rightarrow \tilde{\Delta}} F^{\prime}(\Delta)=\infty, \lim _{\Delta \rightarrow \tilde{\Delta}} \frac{F^{\prime}(\Delta) \Delta}{F(\Delta)}=\infty$ and $\lim _{\Delta \rightarrow \tilde{\Delta}} \frac{F^{\prime}(\Delta) \Delta}{F(\Delta) F(\Delta)}=\frac{(1-1 / R)}{(1 / \Delta)^{2}(1-n)(\Delta-1)^{2}}$. Moreover, $(1-n)\left[u^{\prime}(q)-1\right]=1 / \beta-1$. Accordingly, we get

$$
\Theta(\tilde{q}, R)=1 / \beta-1+\frac{1-\beta R}{\beta R} \frac{R(1-\beta n)^{2}}{(R-1)(1-n)} \frac{u^{\prime \prime}(\tilde{q}) \tilde{q}}{u^{\prime}(\tilde{q})}
$$

Consider first $R \rightarrow 1$. Then we have $\lim _{R \rightarrow 1} \Theta(\tilde{q}, R)=-\infty$. Now consider $R \rightarrow 1 / \beta$. Then we have $\lim _{R \rightarrow 1 / \beta} \Theta(\tilde{q}, R)=1 / \beta-1>0$. Since $\frac{1-\beta R}{\beta} \frac{(1-\beta n)^{2}}{(R-1)(1-n)}$ is monotonically decreasing in $R$, we have a unique critical value $\bar{R}$ such that $\Theta(\tilde{q}, \bar{R})=0$. Thus, if $R<\bar{R}, q=\tilde{q}$ and if $R>\bar{R}, q$ solves $\Theta(q, R)=0$.

Proof of Lemma 4. A stationary equilibrium requires that $M_{+1} / M=\phi / \phi_{+1}=\gamma$. To prove Lemma 4 , note first that using the fact that $\lambda_{\ell}^{k}=u^{\prime}\left(q^{k}\right)\left(1+i_{d}\right)-\left(1+i_{\ell}\right)$, 
$\lambda_{q}^{k}=\lambda_{\ell}^{k}+i_{\ell}$ and $\lambda_{d}^{k}=i_{d}$, the marginal value of money, the marginal value of collateral and the marginal value of private debt in market 3 can be written as follows

$$
\begin{aligned}
V_{m}^{k} & =\beta \phi_{+1}\left(1+i_{d}\right)\left\{1+\left(1-n^{k}\right)\left[u^{\prime}\left(q^{k}\right)-1\right]\right\} \\
V_{b}^{k} & =\beta R\left\{1+\left(1-n^{k}\right)\left[u^{\prime}\left(q^{k}\right) / \Delta-1\right]\right\} \\
V_{y}^{k} & =-\beta \phi_{+1}\left(1+i_{m}\right)\left\{1+\left(1-n^{k}\right)\left[u^{\prime}\left(q^{k}\right) / \Delta-1\right]\right\} .
\end{aligned}
$$

To derive (40) rewrite the first-order condition (27) by using equations (9), (32), and (53)-(55) to get

$$
\frac{1-\beta R}{\beta R}=\sigma^{H}\left(1-n^{H}\right)\left[\frac{u^{\prime}\left(q^{H}\right)}{\Delta}-1\right]+\sigma^{L} \frac{\Delta}{\hat{\Delta}}\left\{\frac{\Delta-\hat{\Delta}}{\Delta}+\left(1-n^{L}\right)\left[u^{\prime}\left(q^{L}\right)-1\right]\right\}
$$

Then, rewrite the first-order condition (26) by using equations (9), (33), (53)-(55) to get

$$
\frac{\gamma-\beta\left(1+i_{d}\right)}{\beta\left(1+i_{d}\right)}=\sigma^{L}\left(1-n^{L}\right)\left[u^{\prime}\left(q^{L}\right)-1\right]+\sigma^{H} \frac{\Delta}{\hat{\Delta}}\left\{\frac{\Delta-\hat{\Delta}}{\Delta}+\left(1-n^{H}\right)\left[\frac{u^{\prime}\left(q^{H}\right)}{\Delta}-1\right]\right\} .
$$

Finally, combine (56) with (57) to get (40).

To derive (41), note that in any equilibrium, the budget constraints in the goods market hold with equality and so

$$
p q^{k}=m^{k}+\ell^{k}=M+y^{k}+\ell^{k}, \quad k=H, L .
$$

Then, use (58) to substitute $y^{H}$ and $y^{L}$ in the money market's market clearing condition (34) and rearrange to get (41).

To derive (42) combine (34) and (58).

Finally, to derive (43) use (40) to write (39) as follows

$$
\frac{R \hat{\Delta}-\Delta}{R \hat{\Delta}(\Delta-1)}=\sigma^{L}\left(1-n^{L}\right) \ell^{L} / M+\sigma^{H}\left(1-n^{H}\right) \ell^{H} / M .
$$

Then, use (58) to substitute $\ell^{H}$ and $\ell^{L}$ and rearrange to get

$$
\frac{R \hat{\Delta}-\Delta}{R \hat{\Delta}(\Delta-1)}=-(1-n)+\frac{1}{z_{m}}\left[\sigma^{L}\left(1-n^{L}\right) q^{L}+\sigma^{H}\left(1-n^{H}\right) q^{H}-\sigma^{L}\left(n^{H}-n^{L}\right) z^{L}\right] .
$$

Finally use (42) and solve for $z_{m}$ to get (42).

Note that equations (56) - (43) must hold in any monetary equilibrium, where agents hold collateral. We now consider the case where no short-selling constraint is binding in the money market to derive (44) and (45). 
When no short-selling constraint is binding in the money market, $\lambda_{m d}^{H}=\lambda_{m \ell}^{L}=0$, and so from (29) $V_{m}^{L}+V_{y}^{L}=V_{m}^{H}+V_{y}^{H}=0$. Then, (53) and (55) imply

$$
u^{\prime}\left(q^{k}\right)=\frac{n^{k}}{1-n^{k}} \frac{(\Delta-\hat{\Delta})}{(\hat{\Delta}-1)}, \quad k=H, L .
$$

Using these expressions to replace $u^{\prime}\left(q^{H}\right)$ and $u^{\prime}\left(q^{L}\right)$ in (56) and solving for $\hat{\Delta}$ yields (44). Finally, to derive (45) use (44) to replace $\hat{\Delta}$ in the above equations.

Proof of Proposition 5. The first thing to note is that the system of equations (40) - (45) can be solved recursively as described in the text. It remains to show under which conditions the short-selling constraints in the money market are non-binding. Thus, we need to verify that $y^{k}<R b /\left[\phi_{+1}\left(1+i_{m}\right)\right]$ and that $m+y^{k}>0$. Using the seller's first-order condition and dividing by $p$, we can write these conditions as follows

$$
z^{k}<\beta R b \hat{\Delta} / \Delta \text { and } z_{m}+z^{k}>0 .
$$

Since $z^{L}>z^{H}$, it is sufficient to check that $z^{L}<\beta R b \hat{\Delta} / \Delta$. Along the same lines, since $z^{L}>z^{H}$, it is sufficient to check that $z^{H}>-z_{m}$.

Let us first consider whether $z^{H}>-z_{m}$. From (42) and (43) $z^{H}>-z_{m}$ if

$$
\sigma^{L}\left(q^{L}-q^{H}\right)<\frac{(\hat{\Delta}-1)\left[\sigma^{L}\left(1-n^{L}\right) q^{L}+\sigma^{H}\left(1-n^{H}\right) q^{H}\right]-\sigma^{L} \sigma^{H}\left(q^{L}-q^{H}\right)\left(n^{H}-n^{L}\right) \hat{\Delta}}{\Phi}
$$

where $\Phi=(R \hat{\Delta}-\Delta) /[R(\Delta-1)]+(1-n) \hat{\Delta}$. Note that $\Phi>(1-n) \hat{\Delta}$ since $R \hat{\Delta}>\Delta$.

Then $n^{H}-n^{L}=\varepsilon$ and $\sigma^{L} n^{L}+\sigma^{H} n^{H}=n$ yield $n^{H}=n+\sigma^{L} \varepsilon$ and $n^{L}=n-\sigma^{H} \varepsilon$. Using these relations and rearranging yields

$$
q^{L}-q^{H}<\frac{(\hat{\Delta}-1)(1-n)\left(\frac{\sigma^{H}}{\sigma^{L}} q^{H}+q^{L}\right)-\varepsilon \sigma^{H}\left(q^{L}-q^{H}\right)}{\Phi} .
$$

Divide by $q^{H}$ and rearrange to get

$$
\frac{q^{L}}{q^{H}}\left[\Phi-(\hat{\Delta}-1)(1-n)+\varepsilon \sigma^{H}\right]<\Phi+\frac{\sigma^{H}}{\sigma^{L}}(\hat{\Delta}-1)(1-n)+\varepsilon \sigma^{H} .
$$

The left-hand side is larger than zero since $\Phi>(1-n) \hat{\Delta}$. Moreover, it is strictly smaller than the right-hand side at $\varepsilon=0$ (since $q^{L}=q^{H}$ at $\varepsilon=0$ ). Then, divide the inequality by $\left[\Phi-(1-n)(\hat{\Delta}-1)+\sigma^{H} \varepsilon\right]$ to get

$$
\frac{q^{L}}{q^{H}}<\frac{\Phi+\frac{\sigma^{H}}{\sigma^{L}}(\hat{\Delta}-1)(1-n)+\sigma^{H} \varepsilon}{\Phi-(\hat{\Delta}-1)(1-n)+\sigma^{H} \varepsilon} .
$$


The left hand side is increasing in $\varepsilon$ and the right-hand side is decreasing. Therefore, there is a unique $\tilde{\varepsilon}_{1}$, such that $z_{H}>-z_{m}$ when $\varepsilon<\tilde{\varepsilon}_{1}$.

We next check $\beta R b \hat{\Delta} / \Delta>z^{L}$. From $\sigma^{H} q^{H}+\sigma^{L} q^{L}=z_{m}+\frac{\beta R b}{\Delta}$, we need $\sigma^{H} q^{H}+$ $\sigma^{L} q^{L}>z_{m}+z^{L} / \hat{\Delta}$, or replacing for $z_{m}$ and $z^{L}$, and rearranging we need

$$
\sigma^{H} q^{H}+\sigma^{L} q^{L}>\frac{\hat{\Delta}\left\{(\hat{\Delta}-1)\left[\sigma^{L}\left(1-n^{L}\right) q^{L}+\sigma^{H}\left(1-n^{H}\right) q^{H}\right]-\left(n^{H}-n^{L}\right) \sigma^{L} \sigma^{H}\left(q^{L}-q^{H}\right) \hat{\Delta}\right\}}{(\hat{\Delta}-1) \Phi}+\frac{\sigma^{H}\left(q^{L}-q^{H}\right)}{(\hat{\Delta}-1)} .
$$

Multiply through by $(\hat{\Delta}-1)$ and arrange to obtain

$$
\left(\sigma^{H} q^{H}+\sigma^{L} q^{L}\right) \hat{\Delta}-q^{L}>\frac{\hat{\Delta}\left\{(\hat{\Delta}-1)\left[\sigma^{L}\left(1-n^{L}\right) q^{L}+\sigma^{H}\left(1-n^{H}\right) q^{H}\right]-\left(n^{H}-n^{L}\right) \sigma^{L} \sigma^{H}\left(q^{L}-q^{H}\right) \hat{\Delta}\right\}}{\Phi} .
$$

Use $n^{H}=n+\sigma^{L} \varepsilon$ and $n^{L}=n-\sigma^{H} \varepsilon$ to substitute $n^{H}$ and $n^{L}$ and rearrange to get

$$
\left(\sigma^{H} q^{H}+\sigma^{L} q^{L}-\frac{q^{L}}{\hat{\Delta}}\right) \Phi>(1-n)\left(\sigma^{L} q^{L}+\sigma^{H} q^{H}\right)(\hat{\Delta}-1)-\sigma^{L} \sigma^{H}\left(q^{L}-q^{H}\right) \varepsilon .
$$

This expression is satisfied at $\varepsilon=0$ since we have $\Phi>(1-n) \hat{\Delta}$. Dividing both sides by $\sigma^{H} q^{H}+\sigma^{L} q^{L}$, and rearranging gives

$$
\frac{\Phi}{\hat{\Delta}\left(\sigma^{H} \frac{q^{H}}{q^{L}}+\sigma^{L}\right)}<\Phi-(\hat{\Delta}-1)(1-n)+\frac{\sigma^{L} \sigma^{H} \varepsilon\left(1-\frac{q^{H}}{q^{L}}\right)}{\sigma^{H} \frac{q^{H}}{q^{L}}+\sigma^{L}} .
$$

Since $\frac{q^{H}}{q^{L}}$ is decreasing in $\varepsilon$, the left-hand side is increasing in $\varepsilon$, and the right-hand side is also increasing in $\varepsilon$. Therefore, given this constraint does not bind at $\varepsilon=0$, either it never binds or it binds for some $\varepsilon>\varepsilon_{1}$. Thus, if $\varepsilon<\varepsilon_{1}=\min \left\{\tilde{\varepsilon}_{1}, \hat{\varepsilon}_{1}\right\}$, a unique equilibrium exists where no short-selling constraint binds.

\section{References}

[1] BIS, 2003. "Committee on Payment and Settlement Systems, Red Book 2003." Bank for International Settlements Publication.

[2] Berentsen, A., G. Camera and C. Waller, 2006. "Money, Credit and Banking." Journal of Economic Theory, forthcoming.

[3] Berentsen, A. and C. J. Waller, 2006. "The Societal Benefits of Outside Versus Inside Bonds." University of Basel working paper. 
[4] Clews, R., 2005. "Implementing monetary policy: reforms to the Bank of England's operations in the money market." Bank of England Quarterly Bulletin, Summer 2005.

[5] ECB, 2005. "Euro Money Market Study 2004." European Central Bank Publication.

[6] ECB, 2004. "The Monetary Policy of the ECB." European Central Bank Publication.

[7] Gaspar, V., G. Quiros, H. Mendizabal, 2004. "Interest rate determination in the interbank market." European Central Bank working paper No. 351.

[8] Goodhart, C.A.E., 1989. "Money, Information and Uncertainty." Macmillan, Second Edition.

[9] Guthrie, G., and J. Wright, 2000. "Open mouth operations." Journal of Monetary Economics 46, 489-516.

[10] Hartmann, P., M. Manna and A. Manzanares, 2001. "The microstructure of the euro money market." Journal of International Money and Finance, Vol. 20 (6), pp. $895-948$.

[11] Heller, D., Y. Lengwiler, 2003. "Payment obligations, reserve requirements, and the demand for central bank balances." Journal of Monetary Economics 50, 419-432.

[12] Kocherlakota, N. 1998. "Money is Memory." Journal of Economic Theory 81, 232-251.

[13] Koeppl, T., C. Monnet and T. Temzelides, 2006. "A Dynamic Model of Settlement," Journal of Economic Theory, forthcoming.

[14] Lagos, R., 2005. "Asset Prices and Liquidity in an Exchange Economy." Federal Reserve Bank of Minneapolis working paper.

[15] Lagos, R. and G. Rocheteau, 2004. "Money and Capital as Competing Media of Exchange." working paper, Federal Reserve Bank of Cleveland. 
[16] Lagos, R. and R. Wright, 2005. "A Unified Framework for Monetary Theory and Policy Analysis." Journal of Political Economy 113, 463-484.

[17] Lester, B., A. Postlewaite and R. Wright, 2006. "What Does Monetary Policy Do?" University of Pennsylvania working paper.

[18] McCandless, G., and Jr. Warren E. Weber, W. (1995). "Some Monetary Facts." Federal Reserve Bank of Minneapolis Quarterly Review Vol. 19, No. 3, Summer 1995, pp. $2-11$

[19] Poole, W., 1968. "Commercial Bank Reserve Management in a Stochastic Model: Implications for Monetary Policy." The Journal of Finance 23, 769-791.

[20] Shi, S. 1997. "A divisible search model of fiat money." Econometrica 65, 75-102.

[21] Telyukova, I. and R. Wright, 2006. "A Model of Money and Credit, with Application to the Credit Card Debt Puzzle." University of Pennsylvania working paper.

[22] Wallace, N., 2001. "Whither Monetary Economics?" International Economic Review 42, 847-869.

[23] Whitesell, W., 2006. "Interest-rate corridors and reserves." Journal of Monetary Economics 53, 1177-1195.

[24] Woodford, M., 2000. "Monetary Policy in a World Without Money," International Finance 3, 229-260.

[25] Woodford, M., 2001. "Monetary Policy in the Information Economy," Mimeo, Princeton University.

[26] Woodford, M., 2003. "Interest and Prices: Foundations of a Theory of Monetary Policy," Princeton University Press. 